Supporting Information

for

\title{
Hydrogen-Bond Synthon Preferences in Cocrystals of Acetazolamide
}

Kaylen J. Meers, ${ }^{\ddagger}$ Thien Nhan Tran, ${ }^{\ddagger}$ Qixuan Zheng, ${ }^{\ddagger}$ Daniel K. Unruh, and Kristin M. Hutchins ${ }^{\star}$

Department of Chemistry \& Biochemistry, Texas Tech University, 1204 Boston Avenue, Lubbock, Texas, 79409, United States.

¥ These authors contributed equally.

*Author email address: kristin.hutchins@ttu.edu

Contents:

1. Materials and cocrystallization experiments

Page S2-S3

2. Single crystal $X$-ray diffraction data

Page S4-S7

3. ${ }^{1} \mathrm{H}$ NMR data for cocrystals

Page S8-S12

4. ${ }^{1} \mathrm{H}$ NMR data for 4,4'-AP before and after purification

Page $\mathrm{S} 13$

5. FTIR spectra of components and cocrystals

Page S14-S18

6. PRXD patterns

Page S19-S22

7. Hirshfeld surface analysis

Page S23-S30

8. X-ray crystal structure

Page S31

9. References

Page S31 


\section{Materials and cocrystallization experiments}

\section{Materials}

Acetazolamide (ACZ) was purchased from Alfa Aesar (Ward Hill, MA, USA). 4,4'-Dipyridyl (BIPY), $N$-Methylpyridin-4-amine (MP4A), and pyrazine (PYZ) were purchased from Oakwood Chemical (Estill, SC, USA). 1,2-Di-(4-pyridyl)-ethane (BPEth) and nicotinamide (NAM) were purchased from Acros Organics (New Jersey, USA). 1,2-Di(4-pyridyl)ethylene (BPE) and 4,4'-Azopyridine (4,4'-AP) were purchased from Sigma-Aldrich Chemical (St. Louis, MO, USA). 2-Aminopyridine (2AP) and 1,3-di(4-pyridyl)propane (BPP) were purchased from Tokyo Chemical Industry (Tokyo, Japan). Acetone and ethanol were purchased from Fisher Scientific (Lenexa, KS, USA).

The 4,4'-AP obtained from the supplier contained impurities (Lot\# MKCJ9359, see Figure S9). Commercial 4,4'-AP was purified by silica gel chromatography with ethyl acetate as the eluant (Figure S10). All other chemicals were used as received.

\section{Cocrystallization experiments:}

Cocrystals of ACZ-BPEth were synthesized by dissolving ACZ (40 mg, $0.18 \mathrm{mmol}$ ) and BPEth (33 $\mathrm{mg}, 0.18 \mathrm{mmol}$ ) in acetone. Slow evaporation of the solution was allowed for a period of 2-3 days until single crystals formed that were appropriate for X-ray diffraction. Cocrystals grown from a $2: 3$ ratio of $A C Z$ ( $40 \mathrm{mg}, 0.18 \mathrm{mmol}$ ) and BPEth (49.5 mg, $0.27 \mathrm{mmol}$ ) in acetone were identical to those grown from a 1:1 ratio.

Cocrystals of ACZ-BPE were synthesized by dissolving ACZ (40 mg, $0.18 \mathrm{mmol}$ ) and BPE (33 $\mathrm{mg}, 0.18 \mathrm{mmol}$ ) in acetone. Slow evaporation of the solution was allowed for a period of 2-3 days until single crystals formed that were appropriate for X-ray diffraction. Cocrystals grown from a $2: 3$ ratio of $\mathbf{A C Z}(40 \mathrm{mg}, 0.18 \mathrm{mmol})$ and $\mathbf{B P E}(49.5 \mathrm{mg}, 0.27 \mathrm{mmol})$ in acetone were identical to those grown from a 1:1 ratio.

Cocrystals of ACZ-BIPY were synthesized by dissolving ACZ (40 mg, $0.18 \mathrm{mmol}$ ) and BIPY (28 $\mathrm{mg}, 0.18 \mathrm{mmol}$ ) in acetone. Slow evaporation of the solution was allowed for a period of 2-3 days until single crystals formed that were appropriate for X-ray diffraction. Cocrystals grown from a $2: 3$ ratio of $\mathbf{A C Z}$ ( $40 \mathrm{mg}, 0.18 \mathrm{mmol}$ ) and BIPY (42 $\mathrm{mg}, 0.27 \mathrm{mmol}$ ) in acetone were identical to those grown from a 1:1 ratio.

Cocrystals of ACZ $4,4^{\prime}$-AP were synthesized by dissolving ACZ (40 mg, $0.18 \mathrm{mmol}$ ) and purified 4,4'-AP (33 mg, $0.18 \mathrm{mmol}$ ) in acetone. Slow evaporation of the solution was allowed for a period of 2-3 days until single crystals formed that were appropriate for X-ray diffraction. Cocrystals grown from a 2:3 ratio of $\mathbf{A C Z}$ (40 mg, $0.18 \mathrm{mmol}$ ) and purified 4,4'-AP (49.5 $\mathrm{mg}, 0.27 \mathrm{mmol})$ in acetone were identical to those grown from a 1:1 ratio. When the non-purified 4,4'-AP (49.5 mg, $0.27 \mathrm{mmol}$ ) was used for cocrystallization with ACZ (40 mg, $0.18 \mathrm{mmol})$ in acetone, a polymorph was obtained (see Table S2 and Figures S20, S35).

Cocrystals of ACZ-BPP were synthesized by dissolving ACZ (40 mg, $0.18 \mathrm{mmol}$ ) and BPP (36 $\mathrm{mg}, 0.18 \mathrm{mmol}$ ) in ethanol. Slow evaporation of the solution was allowed for a period of 2-3 days until single crystals formed that were appropriate for X-ray diffraction. Cocrystals grown from a $2: 3$ ratio of $\mathbf{A C Z}(40 \mathrm{mg}, 0.18 \mathrm{mmol}$ ) and $\mathbf{B P P}(54 \mathrm{mg}, 0.27 \mathrm{mmol})$ in ethanol were identical to those grown from a 1:1 ratio. With the formation of cocrystals of $\mathbf{A C Z} \cdot \mathbf{B P P}, \mathbf{A C Z}$ also crystallized out at the same time. 
Cocrystals of ACZ PYZ were synthesized by dissolving ACZ (40 mg, $0.18 \mathrm{mmol})$ and PYZ (14.6 $\mathrm{mg}, 0.18 \mathrm{mmol}$ ) in acetone. Slow evaporation of the solution was allowed for a period of 2-3 days until single crystals formed that were appropriate for X-ray diffraction. Cocrystals grown from a 2:3 ratio of $\mathbf{A C Z}(40 \mathrm{mg}, 0.18 \mathrm{mmol})$ and PYZ $(25.5 \mathrm{mg}, 0.27 \mathrm{mmol})$ in acetone were identical to those grown from a 1:1 ratio.

Cocrystals of ACZ-2AP were synthesized by dissolving ACZ (40 mg, $0.18 \mathrm{mmol}$ ) and 2 AP (17 $\mathrm{mg}, 0.18 \mathrm{mmol}$ ) in acetone. Slow evaporation of the solution was allowed for a period of 2-3 days until single crystals formed that were appropriate for X-ray diffraction. Cocrystallization experiments using a 1:3 ratio of $\mathbf{A C Z}(40 \mathrm{mg}, 0.18 \mathrm{mmol}$ ) and $2 \mathbf{A P}(51 \mathrm{mg}, 0.54 \mathrm{mmol})$ in acetone yielded solids that were single phases.

Cocrystals of ACZ $\mathbf{M P 4 A}$ were synthesized by dissolving ACZ $(40 \mathrm{mg}, 0.18 \mathrm{mmol})$ and MP4A $(19 \mathrm{mg}, 0.18 \mathrm{mmol}$ ) in ethanol. Slow evaporation of the solution was allowed for a period of 2-3 days until single crystals formed that were appropriate for $\mathrm{X}$-ray diffraction. Cocrystals grown from a 1:3 ratio of $\mathbf{A C Z}(40 \mathrm{mg}, 0.18 \mathrm{mmol}$ ) and MP4A (66 mg, $0.54 \mathrm{mmol})$ in ethanol were identical to those grown from a 1:1 ratio.

Cocrystals of ACZ·NAM were synthesized by dissolving ACZ (40 mg, $0.18 \mathrm{mmol})$ and NAM (22 $\mathrm{mg}, 0.18 \mathrm{mmol}$ ) in acetone. Slow evaporation of the solution was allowed for a period of 2-3 days until single crystals formed that were appropriate for X-ray diffraction. The cocrystal was isostructural to a published cocrystal, but we deposited the structure at $100 \mathrm{~K}$ in the CSD. 


\section{Single-crystal X-ray diffraction data}

X-ray data for ACZ'BPEth, ACZ'BPE, ACZ'BIPY, ACZ'4,4'-AP (alpha polymorph), ACZ'BPP, ACZ-NAM, ACZ'2AP, and ACZ'MP4A were collected using a Bruker PLATFORM three circle diffractometer equipped with an APEX II CCD detector and operated at $1350 \mathrm{~W}(45 \mathrm{kV}, 30 \mathrm{~mA})$ to generate (graphite monochromated) MoKa radiation $(\lambda=0.71073 \AA)$. The crystal sample in oil was mounted on a MiTiGen cryoloop and kept under a cold nitrogen stream throughout the experiment (see tables below for temperatures). Intensity data were corrected for Lorentz, polarization, and background effects using the Bruker program APEX 3. A semi-empirical correction for adsorption was applied using the program SADABS. ${ }^{1}$ The SHELX-2014 ${ }^{2}$ series of programs was used for the solution and refinement of the crystal structure. Hydrogen atoms bound to carbon, nitrogen, and oxygen atoms were located in the difference Fourier map and were geometrically constrained using the appropriate AFIX commands.

Table S1. X-ray data for ACZ'BPEth, ACZ'BPE, and ACZ'BIPY.

\begin{tabular}{|c|c|c|c|}
\hline compound name & ACZ'BPEth & ACZ'BPE & ACZ'BIPY \\
\hline chemical formula & $\mathrm{C}_{20} \mathrm{H}_{24} \mathrm{~N}_{10} \mathrm{O}_{6} \mathrm{~S}_{4}$ & $\mathrm{C}_{20} \mathrm{H}_{24} \mathrm{~N}_{10} \mathrm{O}_{6} \mathrm{~S}_{4}$ & $\mathrm{C}_{9} \mathrm{H}_{10} \mathrm{~N}_{5} \mathrm{O}_{3} \mathrm{~S}_{2}$ \\
\hline formula mass & 628.73 & 626.71 & 300.34 \\
\hline crystal system & Triclinic & Triclinic & Triclinic \\
\hline space group & $P \overline{1}$ & $P \overline{1}$ & $P \overline{1}$ \\
\hline $\mathrm{a} / \mathrm{A}$ & $6.932(3)$ & $7.1085(12)$ & $4.9095(15)$ \\
\hline $\mathrm{b} / \AA ̊$ & $10.374(5)$ & $10.4319(17)$ & $8.172(2)$ \\
\hline$c / \AA ̊$ & $10.886(8)$ & $10.6854(17)$ & $16.493(5)$ \\
\hline$\alpha /^{\circ}$ & $115.758(14)$ & $116.097(2)$ & $98.826(5)$ \\
\hline$\beta /^{\circ}$ & $98.883(17)$ & $101.387(2)$ & $92.894(4)$ \\
\hline $\mathrm{\gamma} /{ }^{\circ}$ & 103.322(12) & 102.011(2) & $104.146(5)$ \\
\hline $\mathrm{V} / \AA^{3}$ & $656.5(7)$ & $657.27(19)$ & $631.4(3)$ \\
\hline$P_{\text {cald }} / \mathrm{g} \mathrm{cm}^{-3}$ & 1.590 & 1.583 & 1.580 \\
\hline $\mathrm{T} / \mathrm{K}$ & $100(2)$ & $180(2)$ & $250(2)$ \\
\hline Z & 1 & 1 & 2 \\
\hline radiation type & MoKa & MoKa & MoKa \\
\hline $\begin{array}{c}\text { absorption coefficient, } \\
\mu / \mathrm{mm}^{-1}\end{array}$ & 0.421 & 0.420 & 0.434 \\
\hline no. of reflections measured & 9395 & 8745 & 11387 \\
\hline $\begin{array}{l}\text { no. of independent } \\
\text { reflections }\end{array}$ & 2654 & 2408 & 2775 \\
\hline no. of reflection $(\mid>2 \sigma(I))$ & 1893 & 2331 & 2423 \\
\hline $\mathrm{R}_{\text {int }}$ & 0.0200 & 0.0519 & 0.0315 \\
\hline $\mathrm{R}_{1}((\mathrm{I}>2 \sigma(\mathrm{I}))$ & 0.0304 & 0.0366 & 0.0342 \\
\hline$w R\left(F^{2}\right)(I>2 \sigma(I))$ & 0.0725 & 0.0833 & 0.0958 \\
\hline $\mathrm{R}_{1}$ (all data) & 0.0356 & 0.0494 & 0.0392 \\
\hline $\mathrm{wR}\left(\mathrm{F}^{2}\right)$ (all data) & 0.0753 & 0.0885 & 0.0999 \\
\hline Goodness-of-fit & 1.060 & 1.032 & 1.050 \\
\hline CCDC deposition number & 1967932 & 1967931 & 1967930 \\
\hline
\end{tabular}


Table S2. X-ray data for: ACZ'4,4'-AP, ACZ'BPP, and ACZ'NAM.

\begin{tabular}{|c|c|c|c|}
\hline compound name & ACZ'4,4'-AP (alpha) & ACZ·BPP & ACZ'NAM \\
\hline chemical formula & $\mathrm{C}_{18} \mathrm{H}_{20} \mathrm{~N}_{12} \mathrm{O}_{6} \mathrm{~S}_{4}$ & $\mathrm{C}_{17} \mathrm{H}_{20} \mathrm{~N}_{6} \mathrm{O}_{3} \mathrm{~S}_{2}$ & $\mathrm{C}_{10} \mathrm{H}_{12} \mathrm{~N}_{6} \mathrm{O}_{4} \mathrm{~S}_{2}$ \\
\hline formula mass & 628.70 & 450.51 & 344.38 \\
\hline crystal system & Monoclinic & Triclinic & Triclinic \\
\hline space group & $P 2_{1} / c$ & $P \overline{1}$ & $P \overline{1}$ \\
\hline$a / \AA$ & $4.7698(6)$ & $7.424(4)$ & $5.1125(7)$ \\
\hline $\mathrm{b} / \AA ̊$ & $17.708(2)$ & $8.602(4)$ & $10.7854(17)$ \\
\hline$c / \AA ̊$ & $15.0852(19)$ & $16.539(8)$ & $13.9440(18)$ \\
\hline$\alpha /^{\circ}$ & 90 & $82.404(13)$ & $70.045(2)$ \\
\hline$\beta /^{\circ}$ & $96.687(2)$ & $78.587(9)$ & $86.636(2)$ \\
\hline $\mathrm{\gamma} /{ }^{\circ}$ & 90 & $78.592(15)$ & $82.391(2)$ \\
\hline $\mathrm{V} / \AA^{3}$ & $1265.5(3)$ & 1010.1(9) & $716.28(18)$ \\
\hline$P_{\text {calc }}\left(\mathrm{g} \mathrm{cm}^{-3}\right)$ & 1.650 & 1.383 & 1.597 \\
\hline $\mathrm{T} / \mathrm{K}$ & $100(2)$ & $100(2)$ & $100(2)$ \\
\hline Z & 2 & 2 & 2 \\
\hline radiation type & MoKa & MoKa & MoKa \\
\hline $\begin{array}{l}\text { absorption coefficient, } \\
\mu / \mathrm{mm}^{-1}\end{array}$ & 0.439 & 0.294 & 0.400 \\
\hline no. of reflections measured & 3244 & 16915 & 12743 \\
\hline $\begin{array}{l}\text { no. of independent } \\
\text { reflections }\end{array}$ & 3244 & 4414 & 3186 \\
\hline no. of reflection $(I>2 \sigma(I))$ & 2717 & 3661 & 2666 \\
\hline $\mathrm{R}_{\text {int }}$ & 0.0669 & 0.0279 & 0.0271 \\
\hline $\mathrm{R}_{1}((\mathrm{I}>2 \sigma(\mathrm{I}))$ & 0.0464 & 0.0365 & 0.0342 \\
\hline$w R\left(F^{2}\right)(I>2 \sigma(I))$ & 0.1316 & 0.0861 & 0.0870 \\
\hline $\mathrm{R}_{1}$ (all data) & 0.0580 & 0.0471 & 0.0424 \\
\hline $\mathrm{wR}\left(\mathrm{F}^{2}\right)$ (all data) & 0.1396 & 0.0912 & 0.0909 \\
\hline Goodness-of-fit & 1.056 & 1.037 & 1.067 \\
\hline CCDC deposition number & 1967928 & 1967933 & 1967934 \\
\hline
\end{tabular}


Table S3. X-ray data for ACZ·2AP and ACZ'MP4A.

\begin{tabular}{|c|c|c|}
\hline compound name & ACZ'2AP & ACZ'MP4A \\
\hline chemical formula & $\mathrm{C}_{13} \mathrm{H}_{18} \mathrm{~N}_{10} \mathrm{O}_{6} \mathrm{~S}_{4}$ & $\mathrm{C}_{10} \mathrm{H}_{14} \mathrm{~N}_{6} \mathrm{O}_{3} \mathrm{~S}_{2}$ \\
\hline formula mass & 538.61 & 330.39 \\
\hline crystal system & Triclinic & Triclinic \\
\hline space group & $P \overline{1}$ & $P \overline{1}$ \\
\hline$a / \AA$ & $10.092(4)$ & $8.498(5)$ \\
\hline $\mathrm{b} / \AA ̊$ & $10.574(4)$ & $9.389(5)$ \\
\hline$c / \AA ̊$ & $10.944(4)$ & $9.932(6)$ \\
\hline$\alpha /^{\circ}$ & $92.064(5)$ & $64.176(13)$ \\
\hline$\beta /^{\circ}$ & $115.146(5)$ & $88.605(13)$ \\
\hline $\mathrm{Y}^{\circ}$ & $96.892(5)$ & $83.290(17)$ \\
\hline $\mathrm{V} / \AA^{3}$ & $1044.5(7)$ & $708.1(7)$ \\
\hline$P_{\text {calc }}\left(\mathrm{g} \mathrm{cm}^{-3}\right)$ & 1.712 & 1.550 \\
\hline $\mathrm{T} / \mathrm{K}$ & $100(2)$ & $100(2)$ \\
\hline $\mathrm{Z}$ & 2 & 2 \\
\hline radiation type & MoKa & MoKa \\
\hline $\begin{array}{c}\text { absorption coefficient, } \\
\mu / \mathrm{mm}^{-1}\end{array}$ & 0.513 & 0.396 \\
\hline no. of reflections measured & 18144 & 11837 \\
\hline $\begin{array}{l}\text { no. of independent } \\
\text { reflections }\end{array}$ & 4631 & 3144 \\
\hline no. of reflection $(I>2 \sigma(I))$ & 3878 & 2804 \\
\hline $\mathrm{R}_{\text {int }}$ & 0.0365 & 0.0268 \\
\hline $\mathrm{R}_{1}((\mathrm{I}>2 \sigma(\mathrm{l}))$ & 0.0354 & 0.0327 \\
\hline$w R\left(F^{2}\right)(I>2 \sigma(I))$ & 0.0841 & 0.0854 \\
\hline $\mathrm{R}_{1}$ (all data) & 0.0438 & 0.0368 \\
\hline $\mathrm{wR}\left(\mathrm{F}^{2}\right)$ (all data) & 0.0881 & 0.0882 \\
\hline Goodness-of-fit & 1.029 & 1.019 \\
\hline CCDC deposition number & 1967927 & 1967929 \\
\hline
\end{tabular}


X-ray data for ACZ'PYZ and ACZ'4,4'-AP (beta polymorph) was collected on a Rigaku XtaLAB Synergy- $i$ Kappa diffractometer equipped with a HyPix-6000HE HPC detector and a PhotonJet- $i$ $X$-ray source operated at $50 \mathrm{~W}(50 \mathrm{kV}, 1 \mathrm{~mA})$ to generate $\mathrm{Cu}$ Ka radiation $(\lambda=1.54178 \AA)$. Crystals were transferred from the vial and placed on a glass slide in polyisobutylene. The crystal was glued to a MîTiGen cryoloop and transferred to the instrument where it was analyzed. The sample was optically centered with the aid of a video camera to insure that no translations were observed as the crystal was rotated through all positions. Intensity data were corrected for Lorentz, polarization, and background effects using CrysAlis ${ }^{\text {Pro }}{ }^{3}$ A numerical absorption correction was applied based on a Gaussian integration over a multifaceted crystal and followed by a semiempirical correction for adsorption applied using the program SCALE3 ABSPACK. ${ }^{4}$ The SHELX$2014^{2}$ series of programs was used for the solution and refinement of the crystal structure. Hydrogen atoms bound to carbon atoms were located in the difference Fourier map and were geometrically constrained using the appropriate AFIX commands.

Table S4. X-ray data for ACZ·PYZ.

\begin{tabular}{|c|c|c|}
\hline compound name & ACZ:PYZ & ACZ'4,4'-AP (beta) \\
\hline chemical formula & $\mathrm{C}_{6} \mathrm{H}_{8} \mathrm{~N}_{5} \mathrm{O}_{3} \mathrm{~S}_{2}$ & $\mathrm{C}_{9} \mathrm{H}_{10} \mathrm{~N}_{6} \mathrm{O}_{3} \mathrm{~S}_{2}$ \\
\hline formula mass & 262.29 & 314.35 \\
\hline crystal system & Triclinic & Triclinic \\
\hline space group & $P \overline{1}$ & $P \overline{1}$ \\
\hline $\mathrm{a} / \AA$ & $5.28380(10)$ & $7.02350(10)$ \\
\hline $\mathrm{b} / \AA$ & $9.6006(2)$ & $10.43170(10)$ \\
\hline $\mathrm{c} / \AA$ & $10.8847(2)$ & $10.54300(10)$ \\
\hline$\alpha /^{\circ}$ & $100.4600(10)$ & $117.2650(10)$ \\
\hline$\beta /^{\circ}$ & $99.8180(10)$ & $101.4890(10)$ \\
\hline $\mathrm{\gamma} /{ }^{\circ}$ & $100.2740(10)$ & $99.3130(10)$ \\
\hline $\mathrm{V} / \AA^{3}$ & $522.395(18)$ & $643.851(14)$ \\
\hline $\mathrm{P}_{\text {cald }} / \mathrm{g} \mathrm{cm}^{-3}$ & 1.668 & 1.621 \\
\hline $\mathrm{T} / \mathrm{K}$ & $100(2)$ & $100(2)$ \\
\hline Z & 2 & 2 \\
\hline radiation type & $\mathrm{Cu} \mathrm{Ka}$ & $\mathrm{Cu} \mathrm{Ka}$ \\
\hline $\begin{array}{c}\text { absorption coefficient, } \\
\mu / \mathrm{mm}^{-1}\end{array}$ & 4.694 & 3.974 \\
\hline no. of reflections measured & 19130 & 15177 \\
\hline $\begin{array}{l}\text { no. of independent } \\
\text { reflections }\end{array}$ & 2149 & 2621 \\
\hline no. of reflection $(1>2 \sigma(I))$ & 2075 & 2460 \\
\hline $\mathrm{R}_{\text {int }}$ & 0.0434 & 0.0480 \\
\hline $\mathrm{R}_{1}((\mathrm{I}>2 \sigma(\mathrm{I}))$ & 0.0277 & 0.0315 \\
\hline$w R\left(F^{2}\right)(I>2 \sigma(I))$ & 0.0700 & 0.0836 \\
\hline $\mathrm{R}_{1}$ (all data) & 0.0284 & 0.0332 \\
\hline$w R\left(F^{2}\right)$ (all data) & 0.0706 & 0.0851 \\
\hline Goodness-of-fit & 1.101 & 1.092 \\
\hline CCDC deposition number & 1967935 & 2012096 \\
\hline
\end{tabular}




\section{3. ${ }^{1} \mathrm{H}$ NMR data for cocrystals}

Single crystals from the cocrystallization experiments were removed from the vial and dissolved in DMSO- $d_{6}$ for NMR experiments. NMR data was collected using a JOEL ECS $400 \mathrm{MHZ}$ Spectrometer with multinuclear, direct and inverse detection probes, automatic sample changer, variable temperature, and Z-gradient capabilities. The instrument was purchased with funds granted from National Science Foundation grant number CHE-1048553.

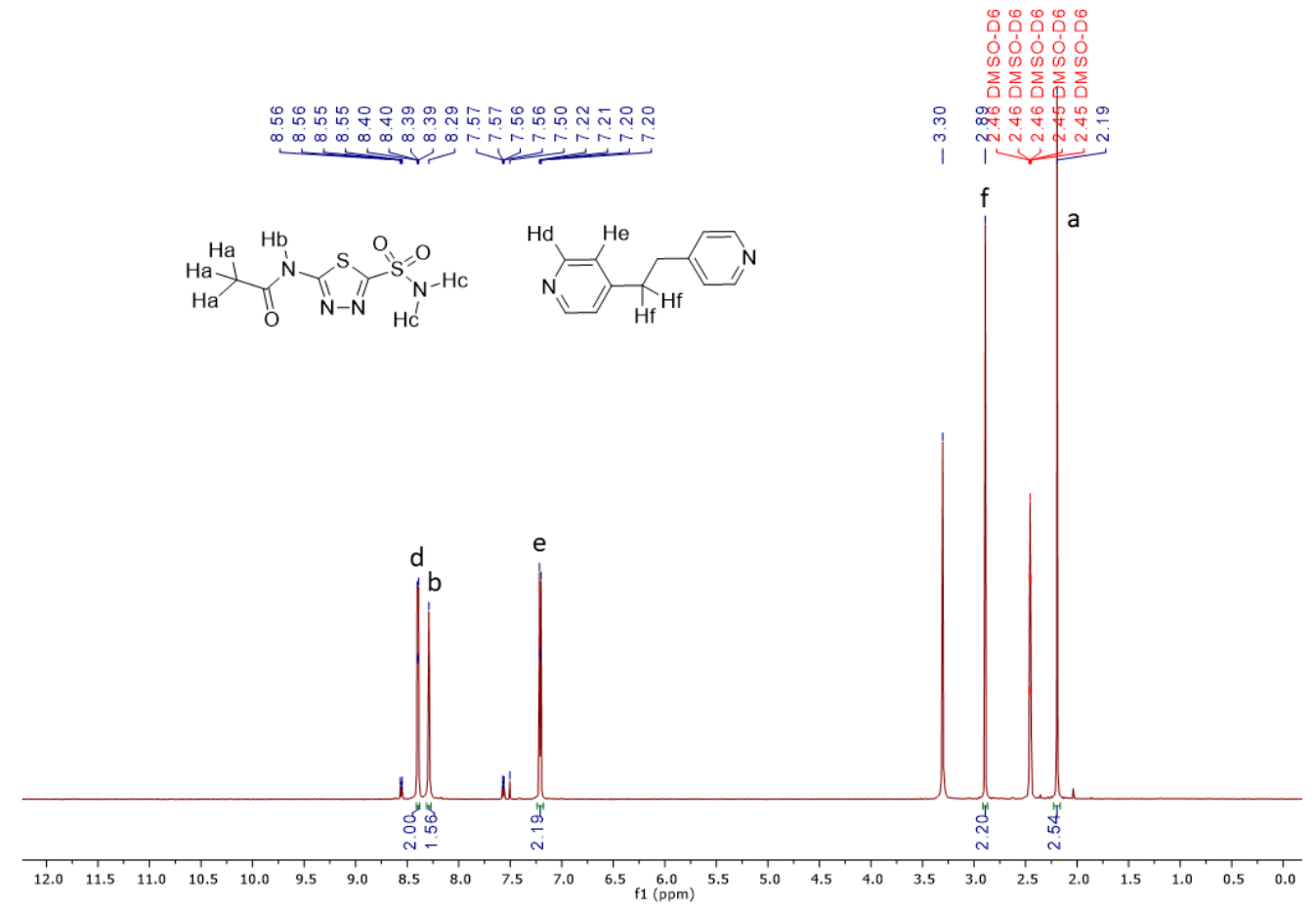

Figure S1. ${ }^{1} \mathrm{H}$ NMR spectrum of ACZ·BPEth. 


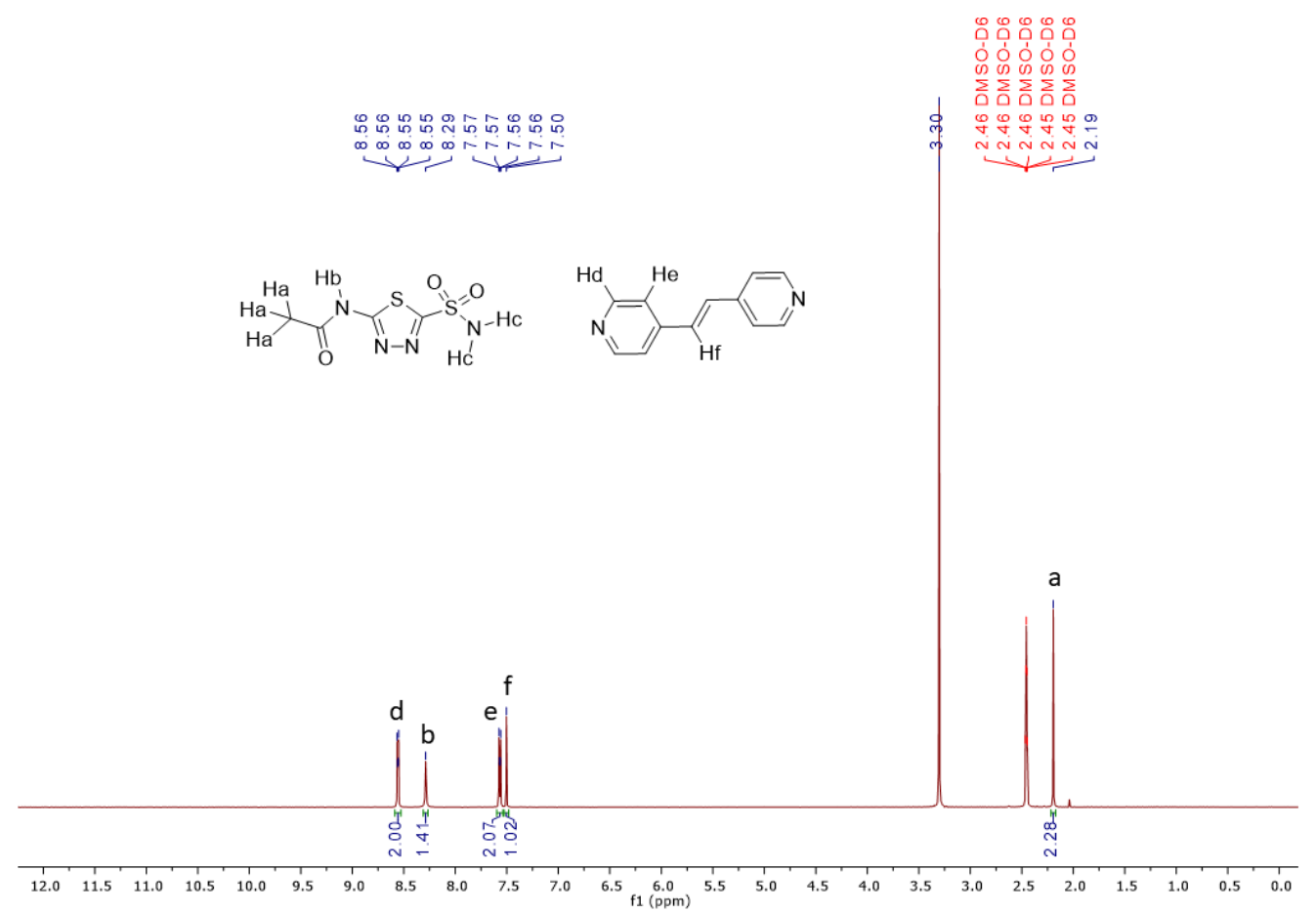

Figure S2. ${ }^{1} \mathrm{H}$ NMR spectrum of ACZ-BPE.

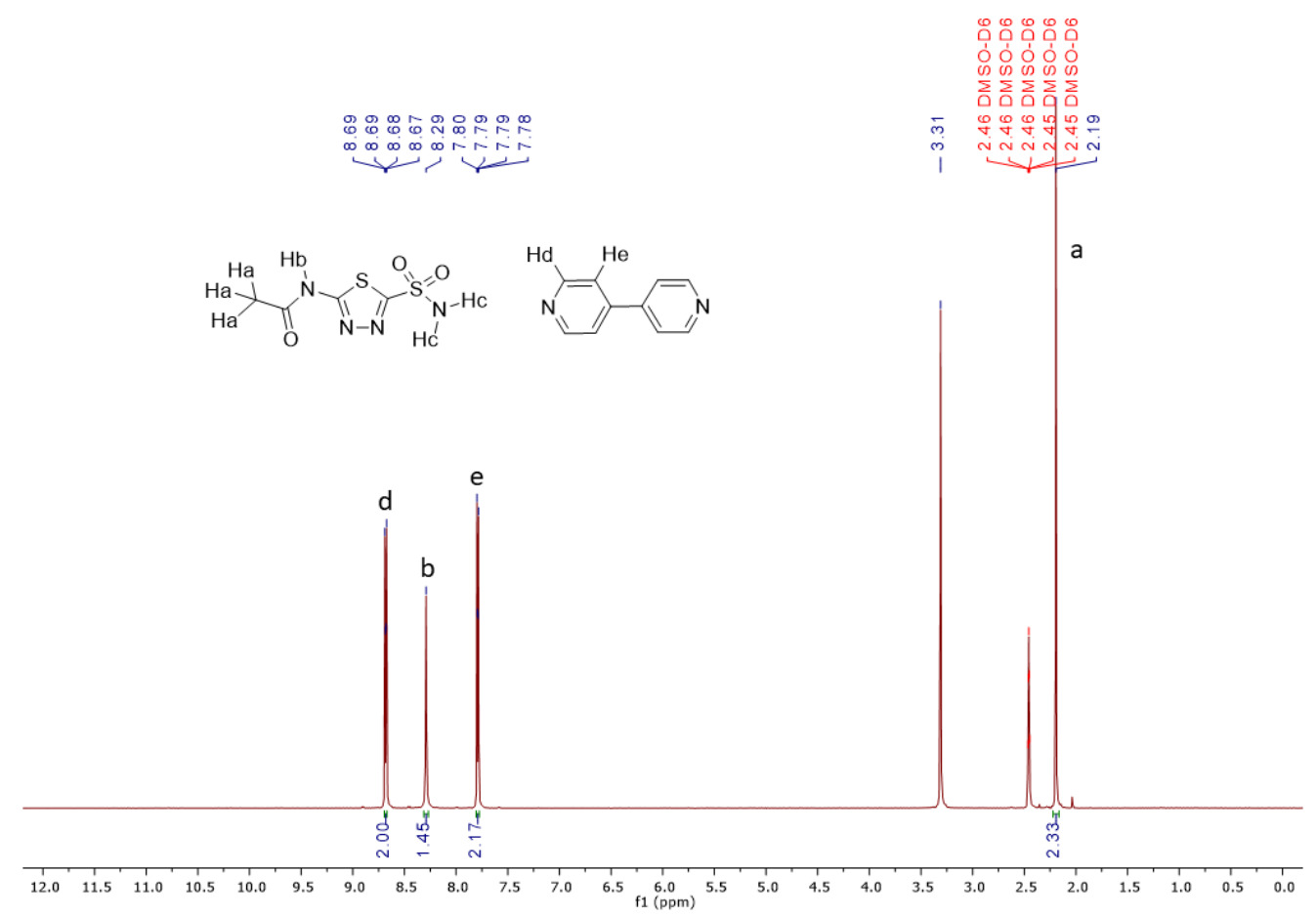

Figure S3. ${ }^{1} \mathrm{H}$ NMR spectrum of ACZ-4,4'-BIPY. 


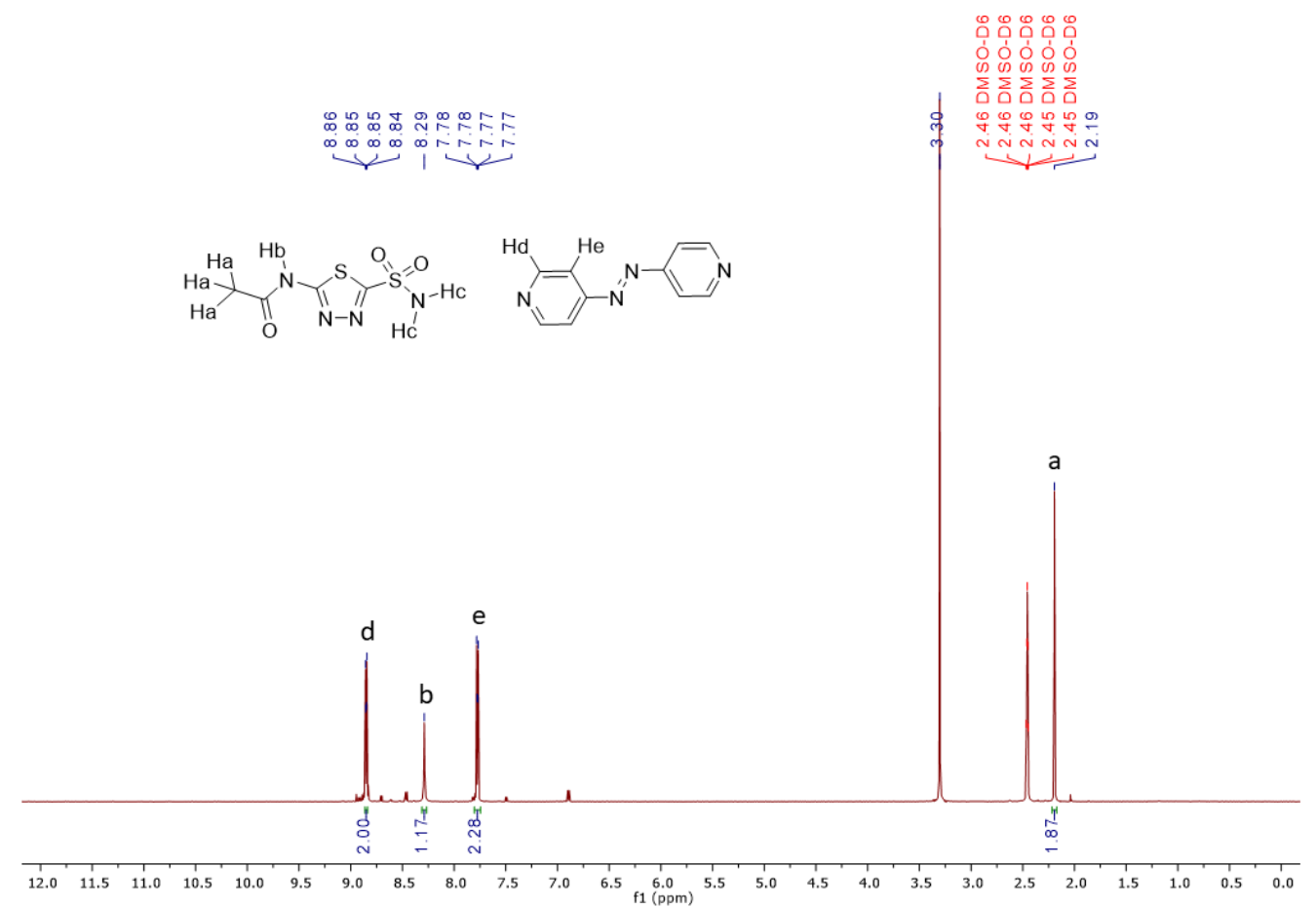

Figure S4. ${ }^{1} \mathrm{H}$ NMR spectrum of ACZ-4,4'-AP.

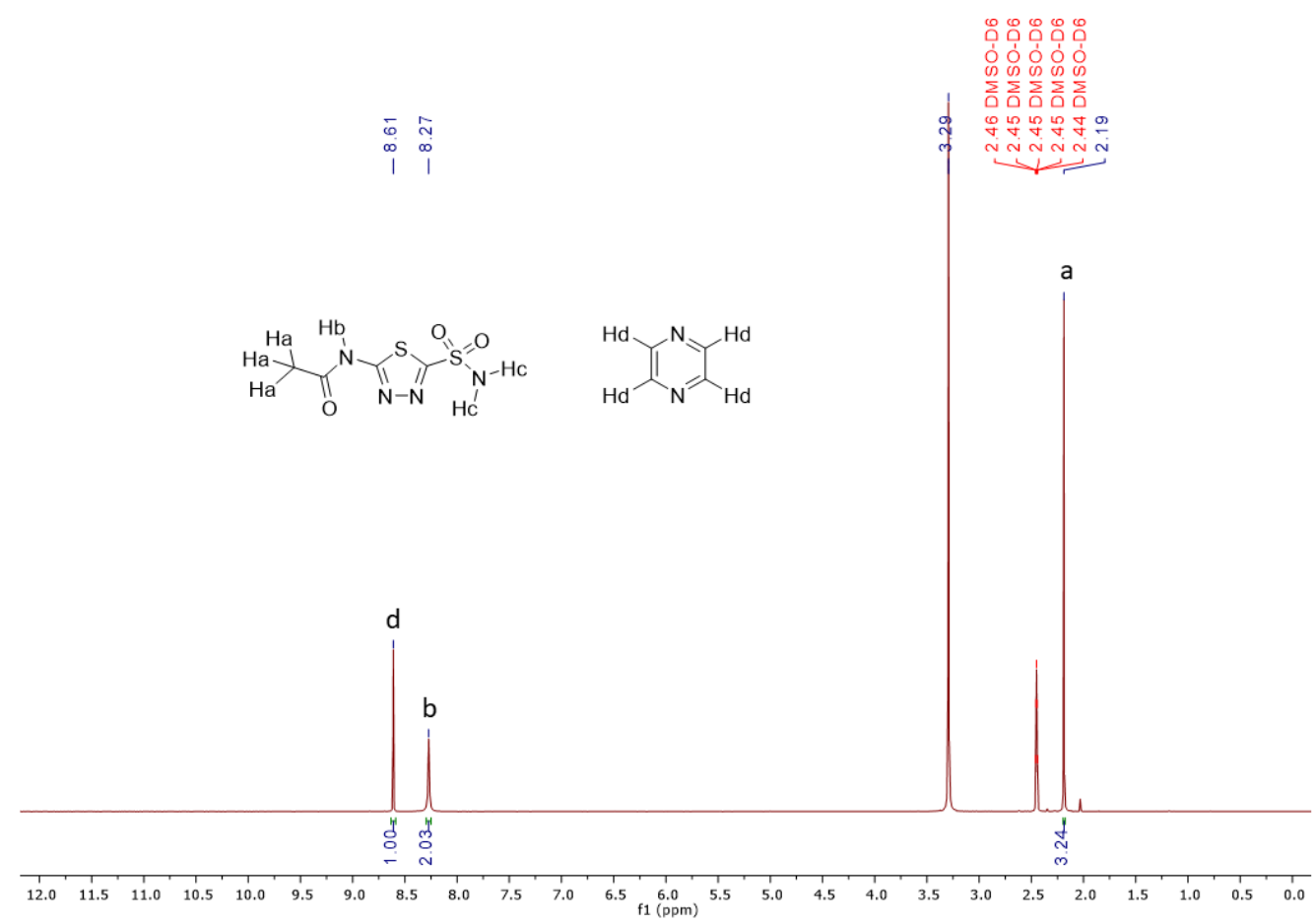

Figure S5. ${ }^{1} \mathrm{H}$ NMR spectrum of ACZ.PYZ. 

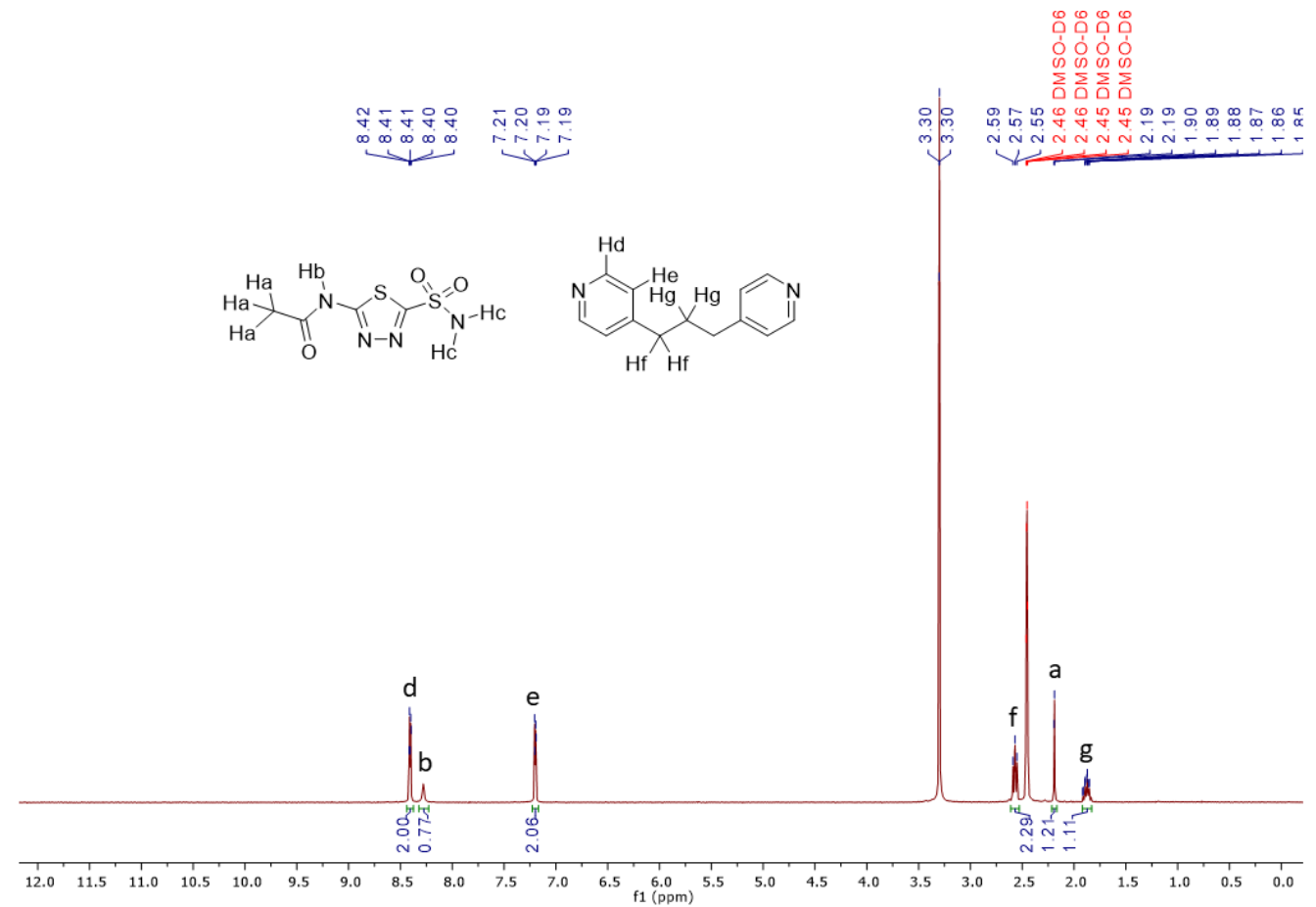

Figure S6. ' $\mathrm{H}$ NMR spectrum of ACZ-BPP.
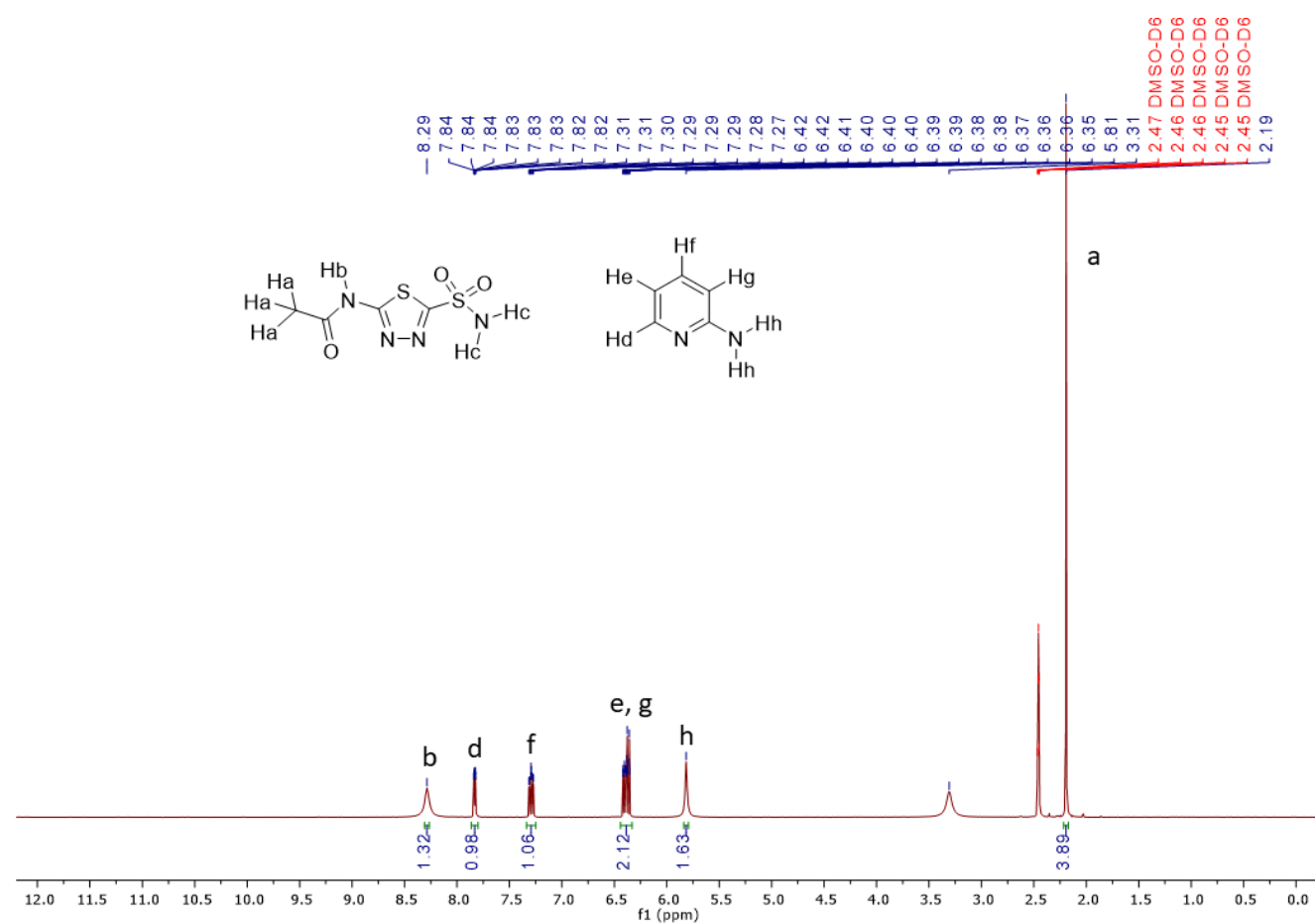

Figure S7. ${ }^{1} \mathrm{H}$ NMR spectrum of ionic cocrystal ACZ-2AP. 


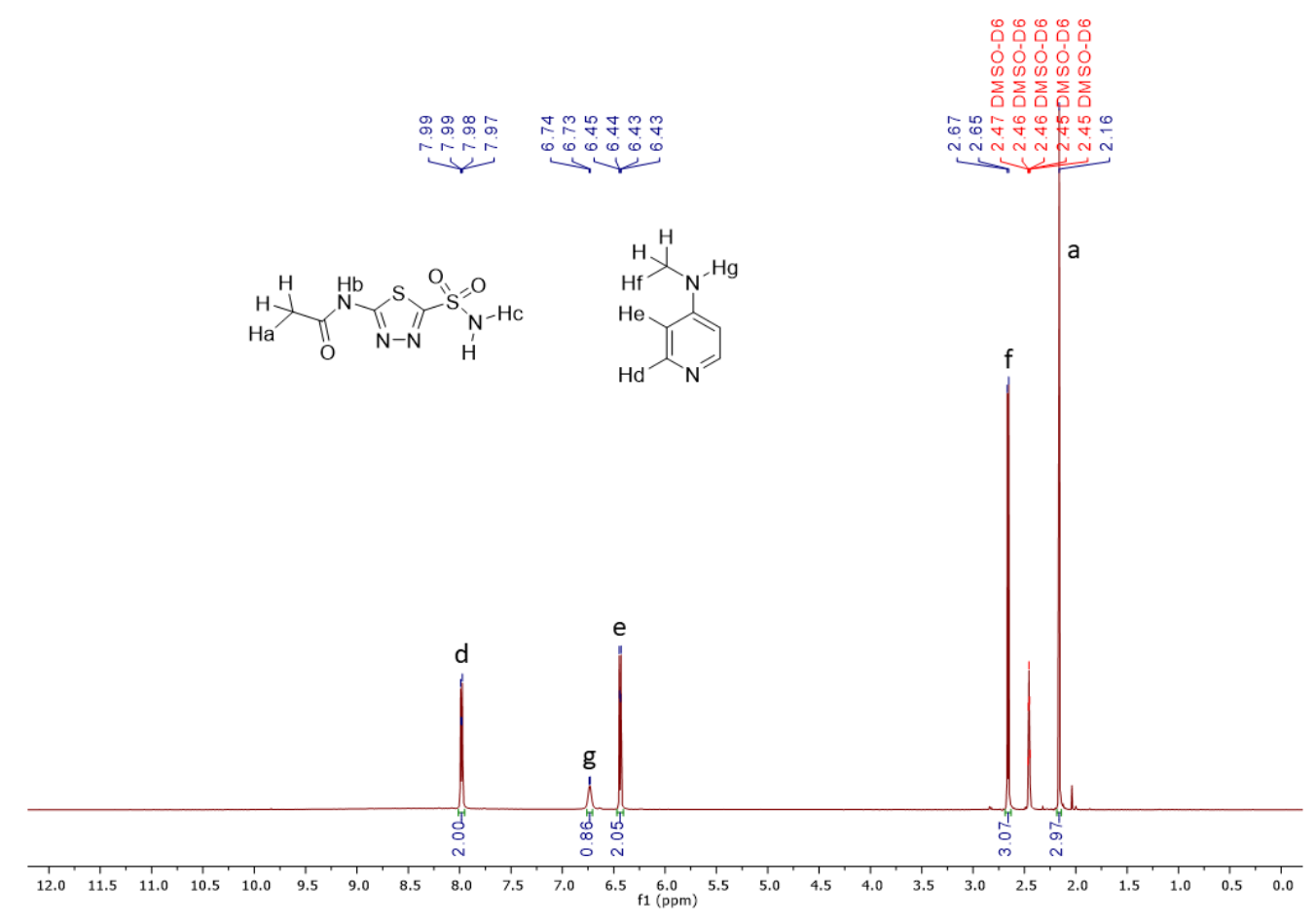

Figure S8. ${ }^{1} \mathrm{H}$ NMR spectrum of ionic cocrystal ACZ-MP4A. 
4. ${ }^{1} \mathrm{H}$ NMR data for 4,4 '-AP before and after purification

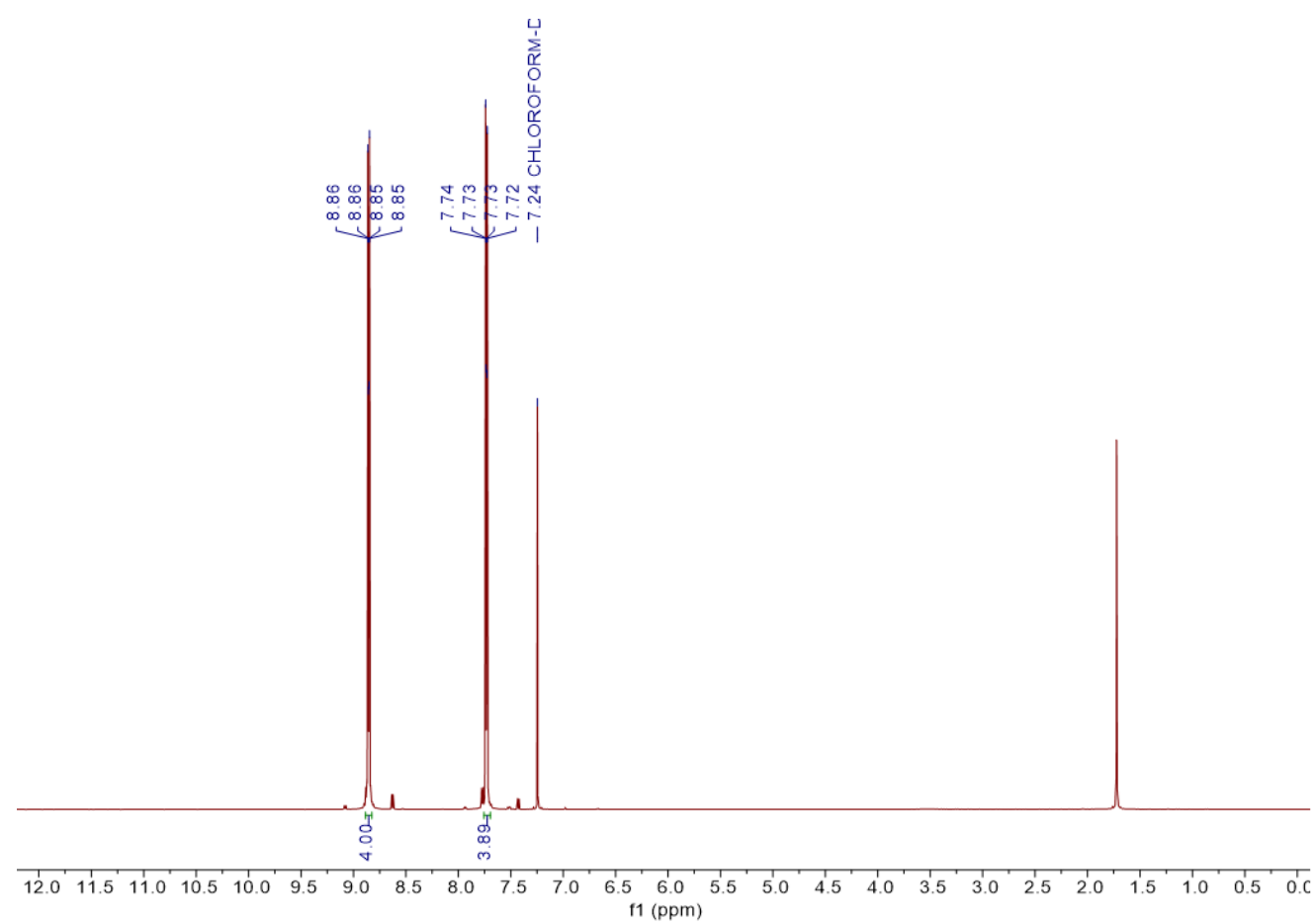

Figure S9. ${ }^{1} \mathrm{H}$ NMR spectrum of 4,4'-AP from supplier, before purification.

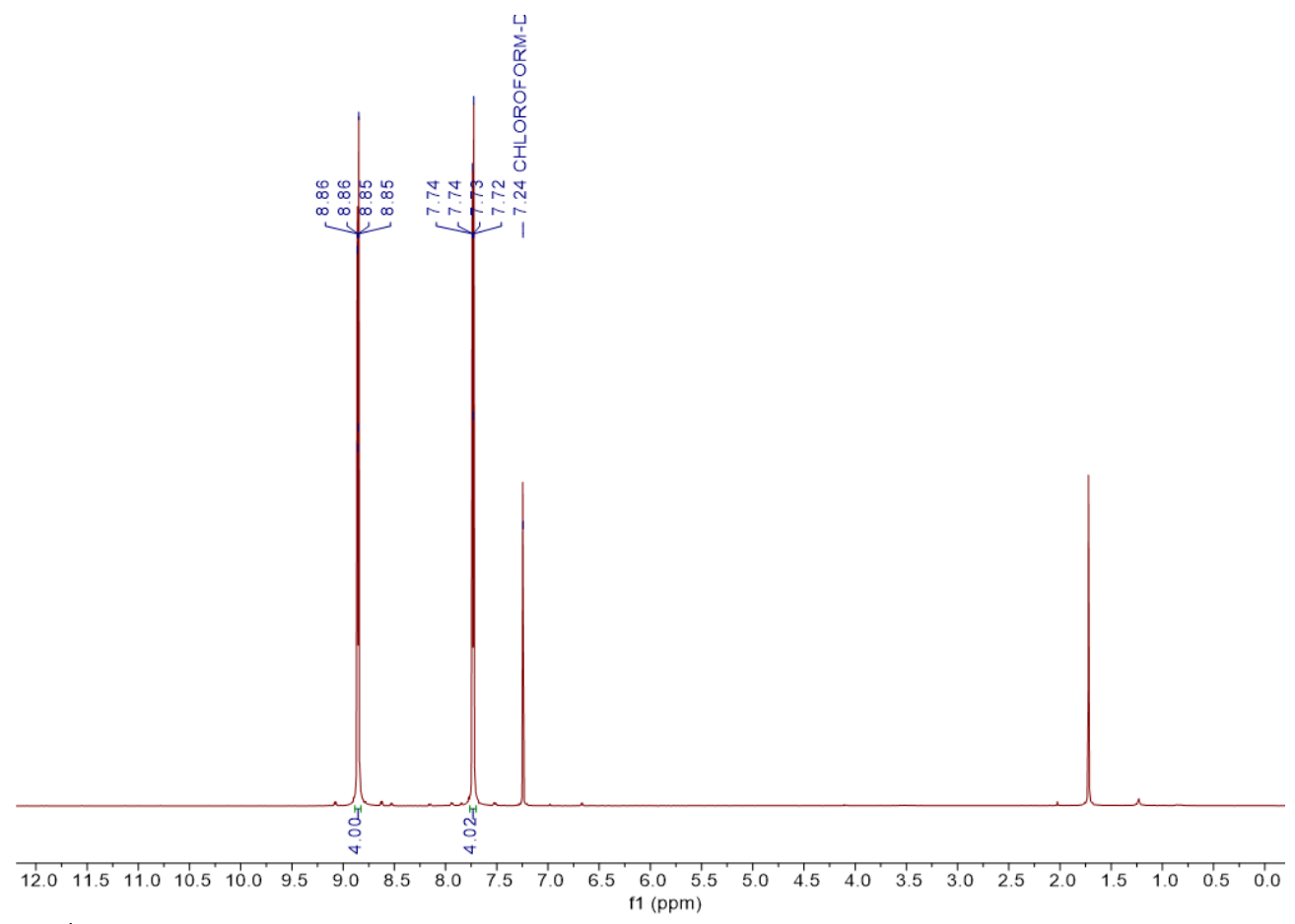

Figure S10. ${ }^{1} \mathrm{H}$ NMR spectrum of 4,4'-AP after purification. 


\section{FTIR spectra of components and cocrystals}

FTIR spectra were recorded on a Nicolet iS10 infrared spectrometer using an ATR attachment equipped with a diamond crystal.

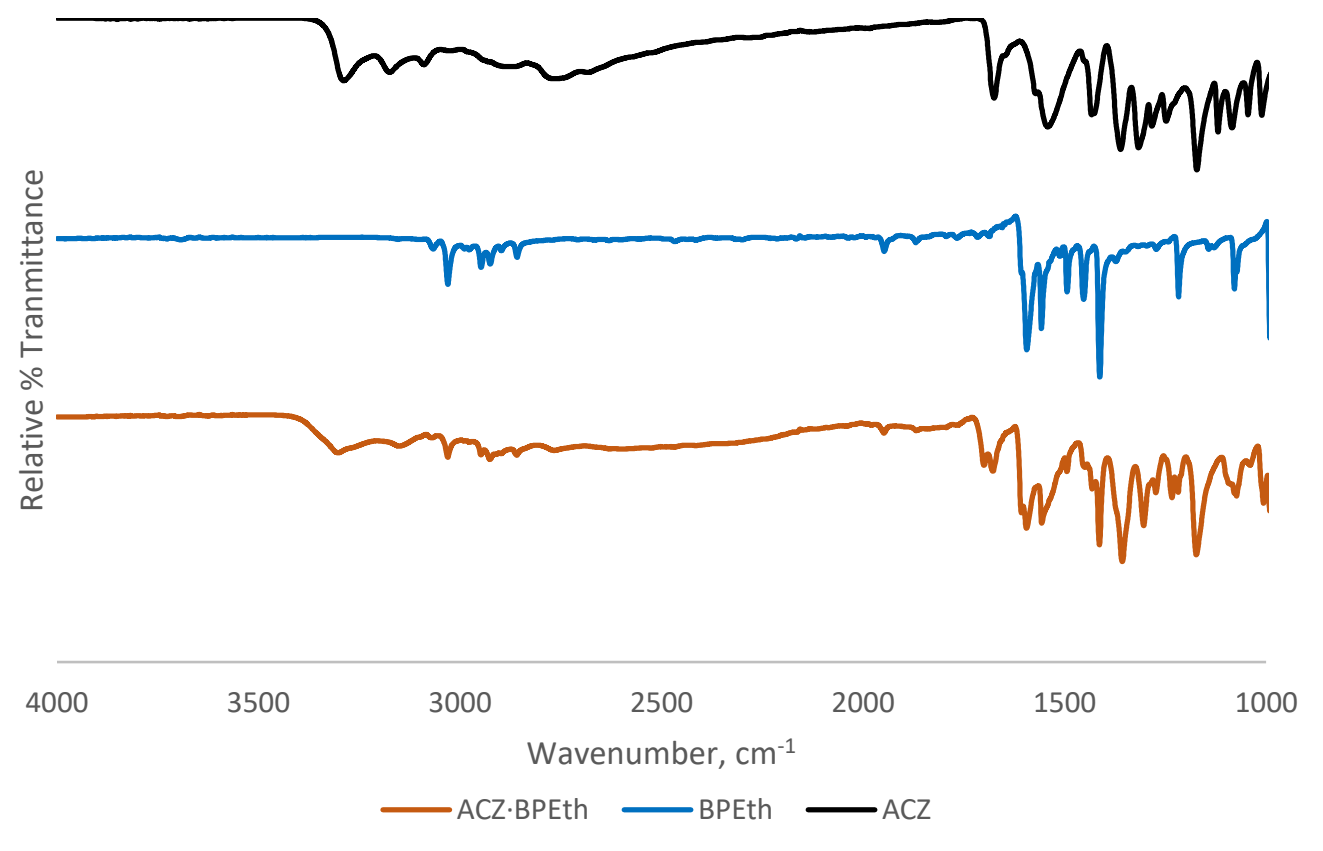

Figure S11. FTIR spectra of ACZ, BPEth, and ACZ·BPEth.

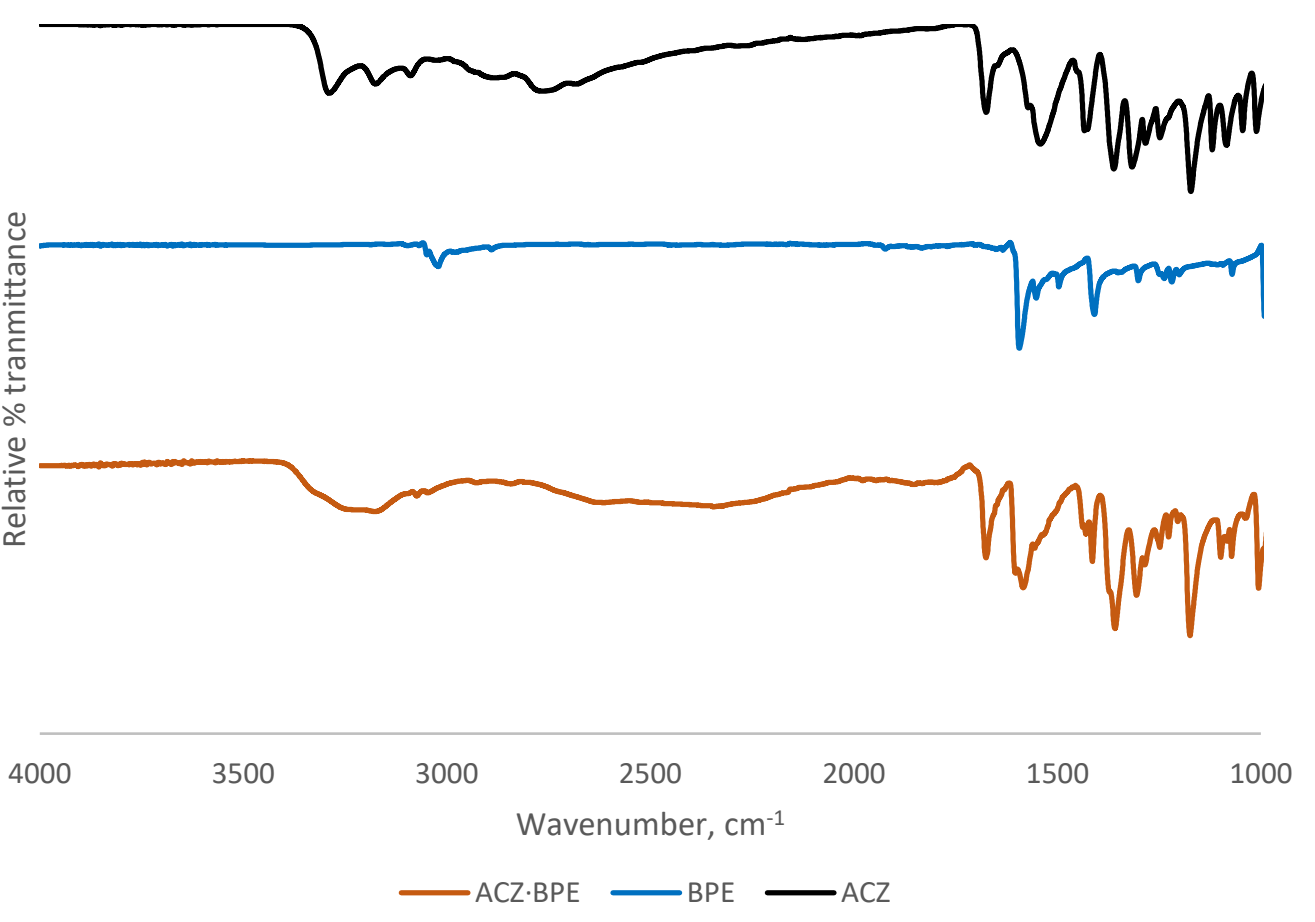

Figure S12. FTIR spectra of ACZ, BPE, and ACZ·BPE. 


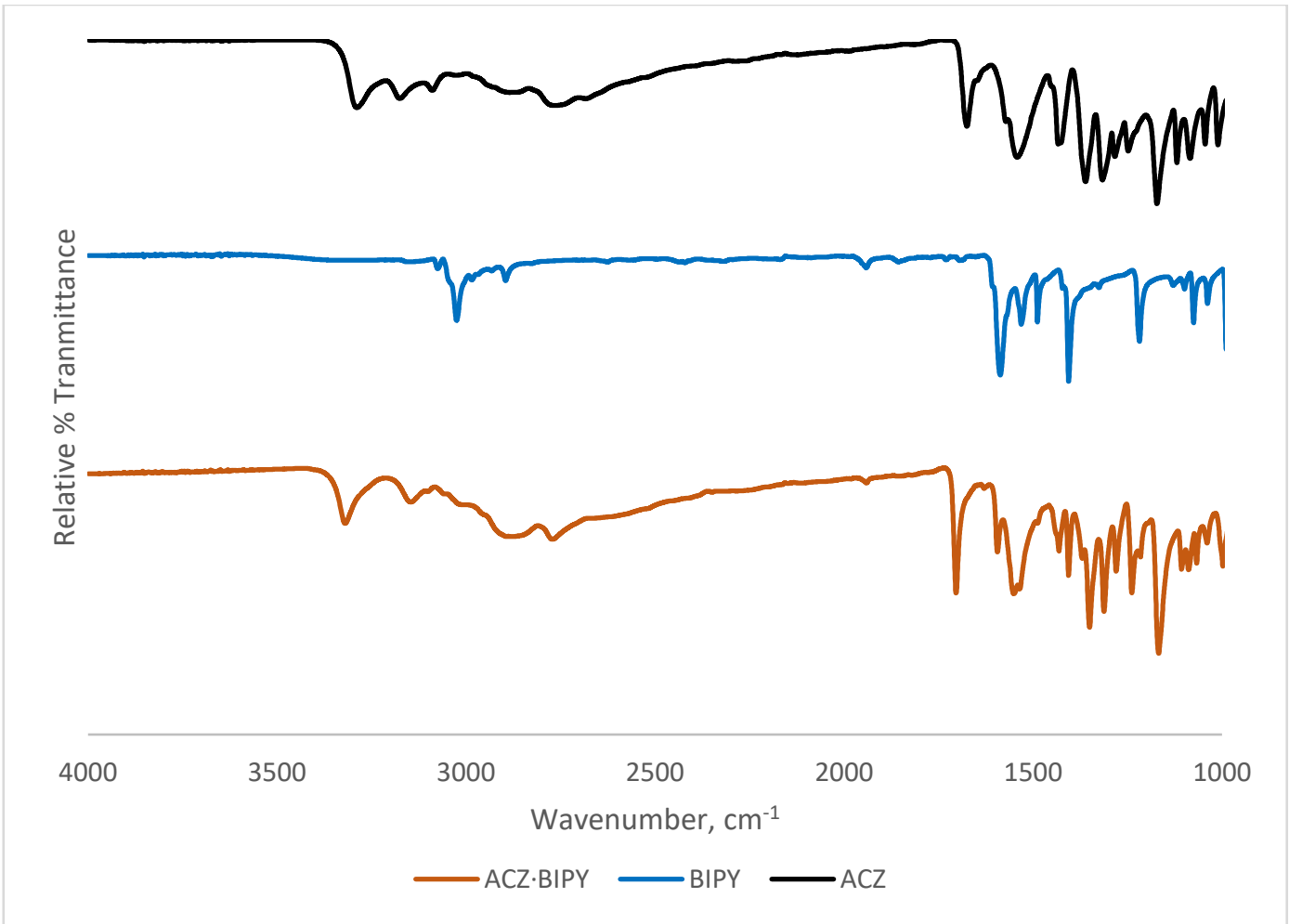

Figure S13. FTIR spectra of ACZ, 4,4'-BIPY, and ACZ-4,4'-BIPY.

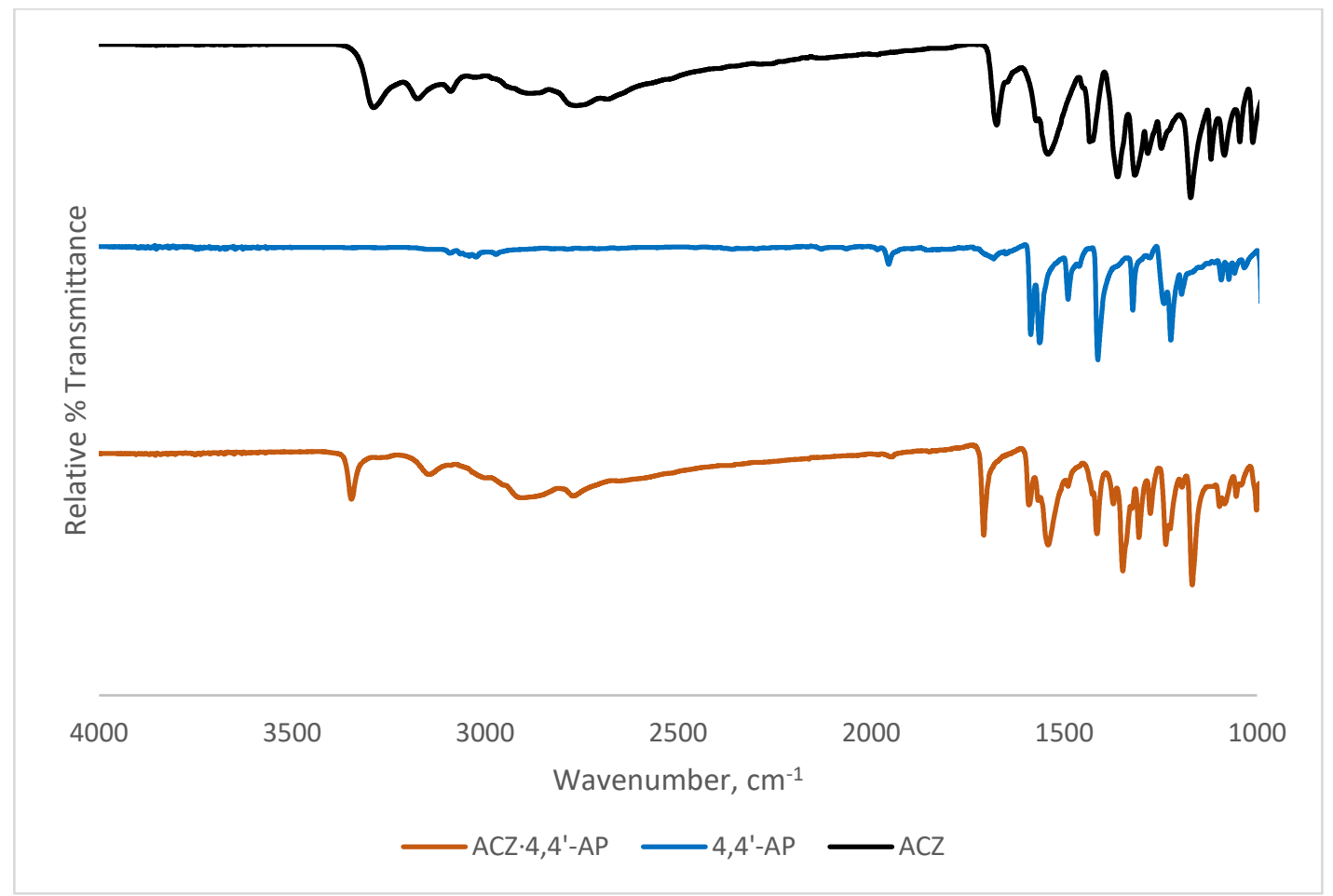

Figure S14. FTIR spectra of ACZ, purified 4,4'-AP, and ACZ-4,4'-AP. 


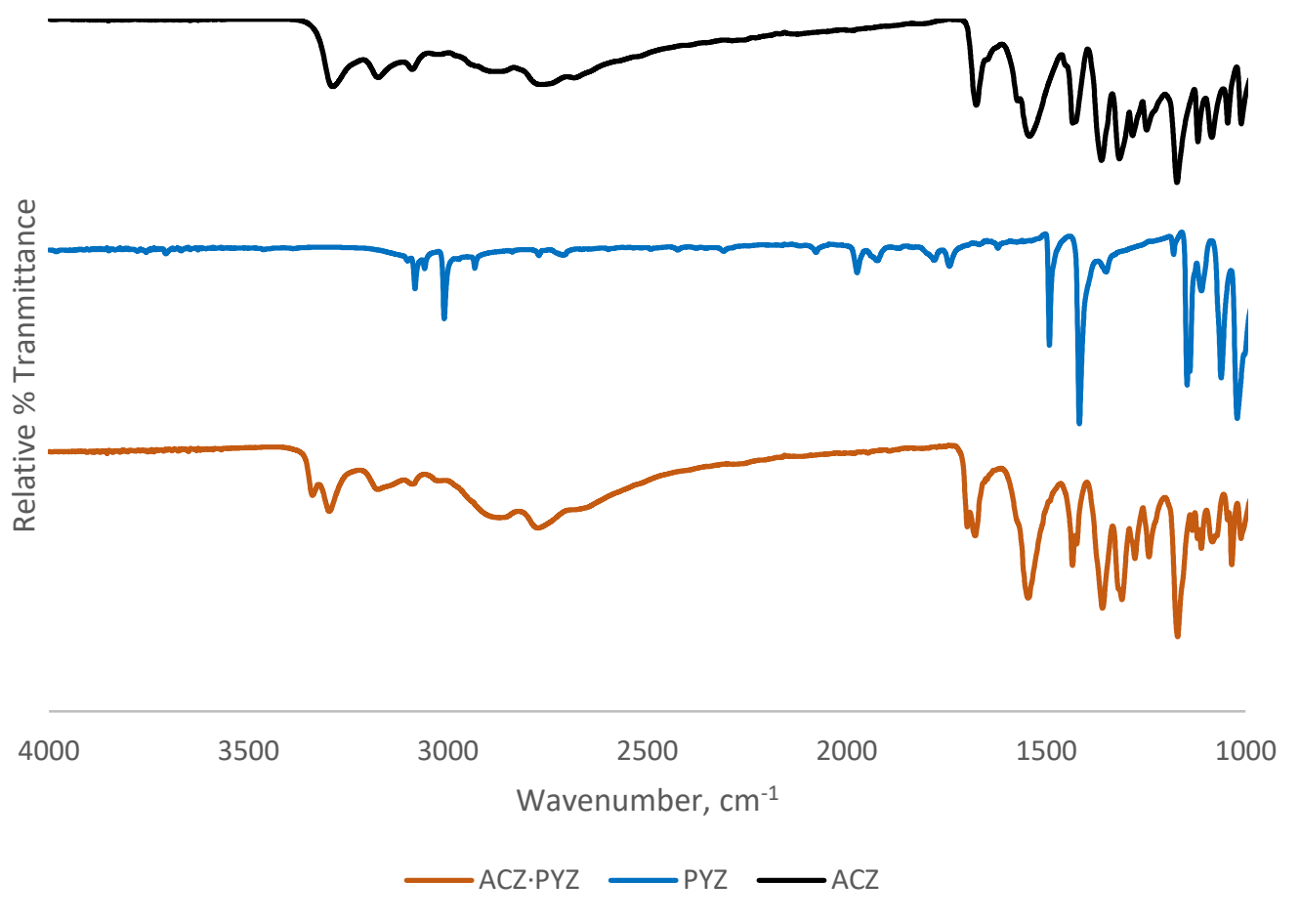

Figure S15. FTIR spectrum of ACZ, PYZ, and ACZ.PYZ.

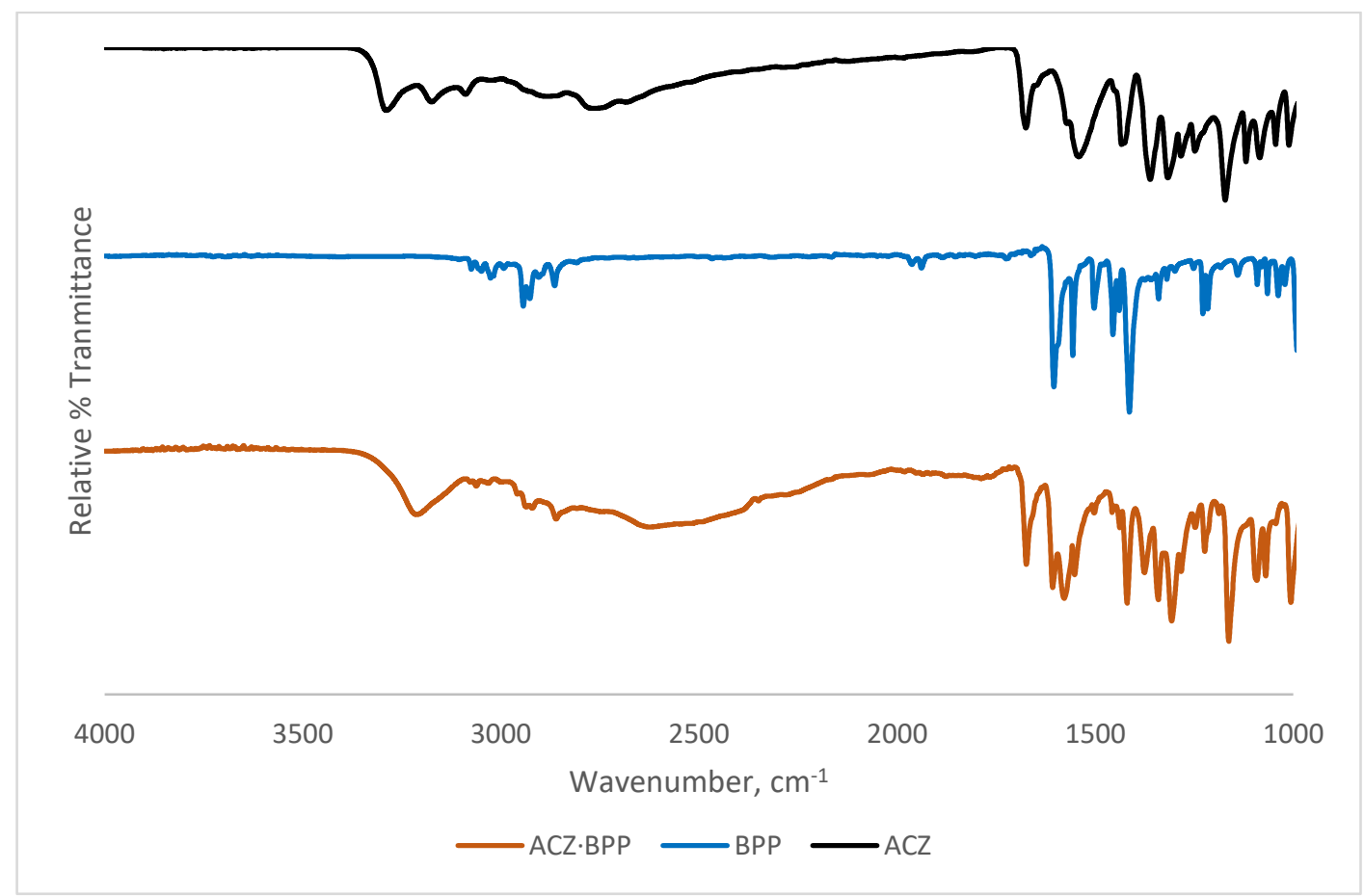

Figure S16. FTIR spectra of ACZ, BPP, and ACZ'BPP. 


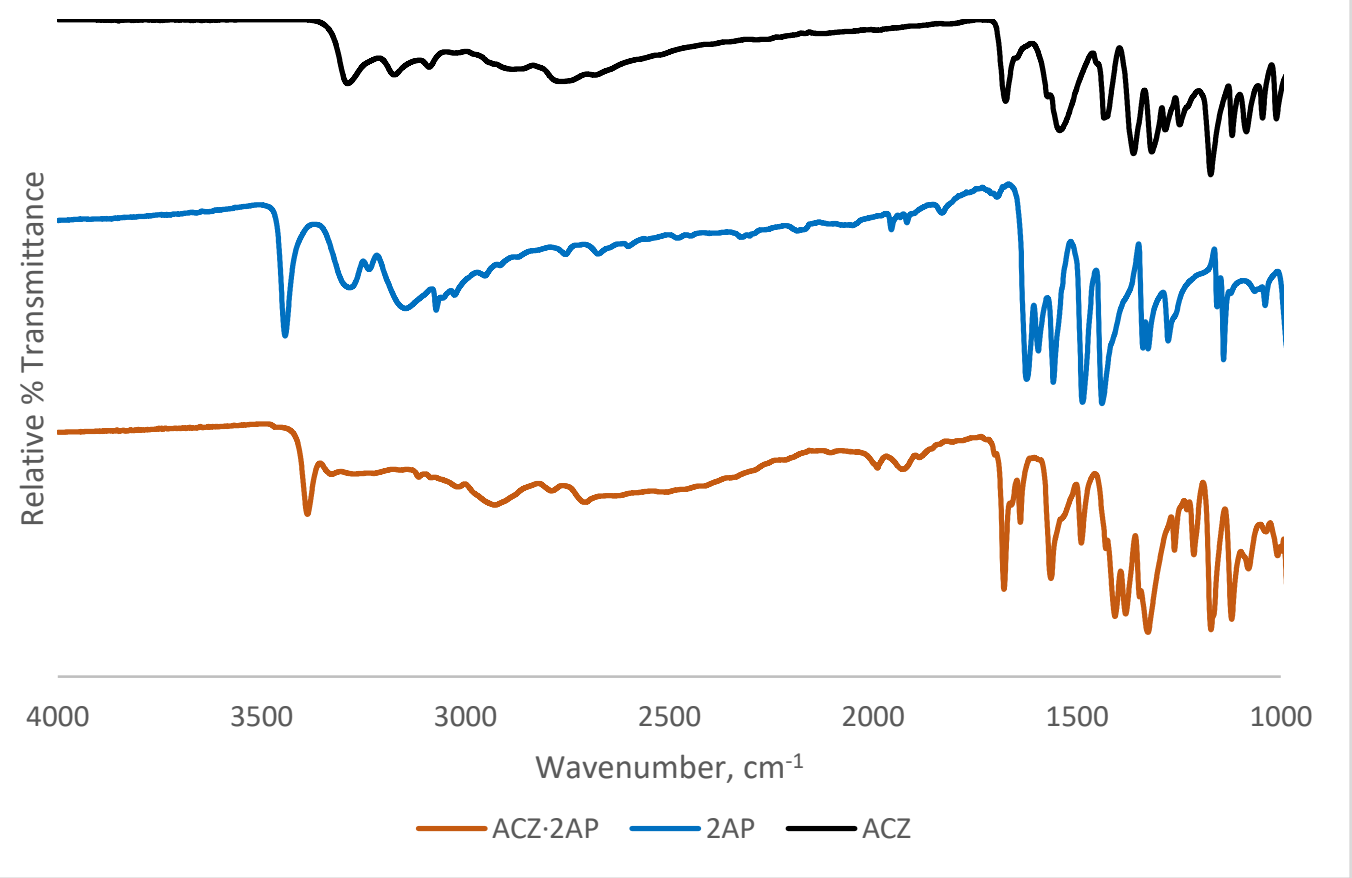

Figure S17. FTIR spectra of ACZ, 2AP, and ionic cocrystal ACZ.2AP.

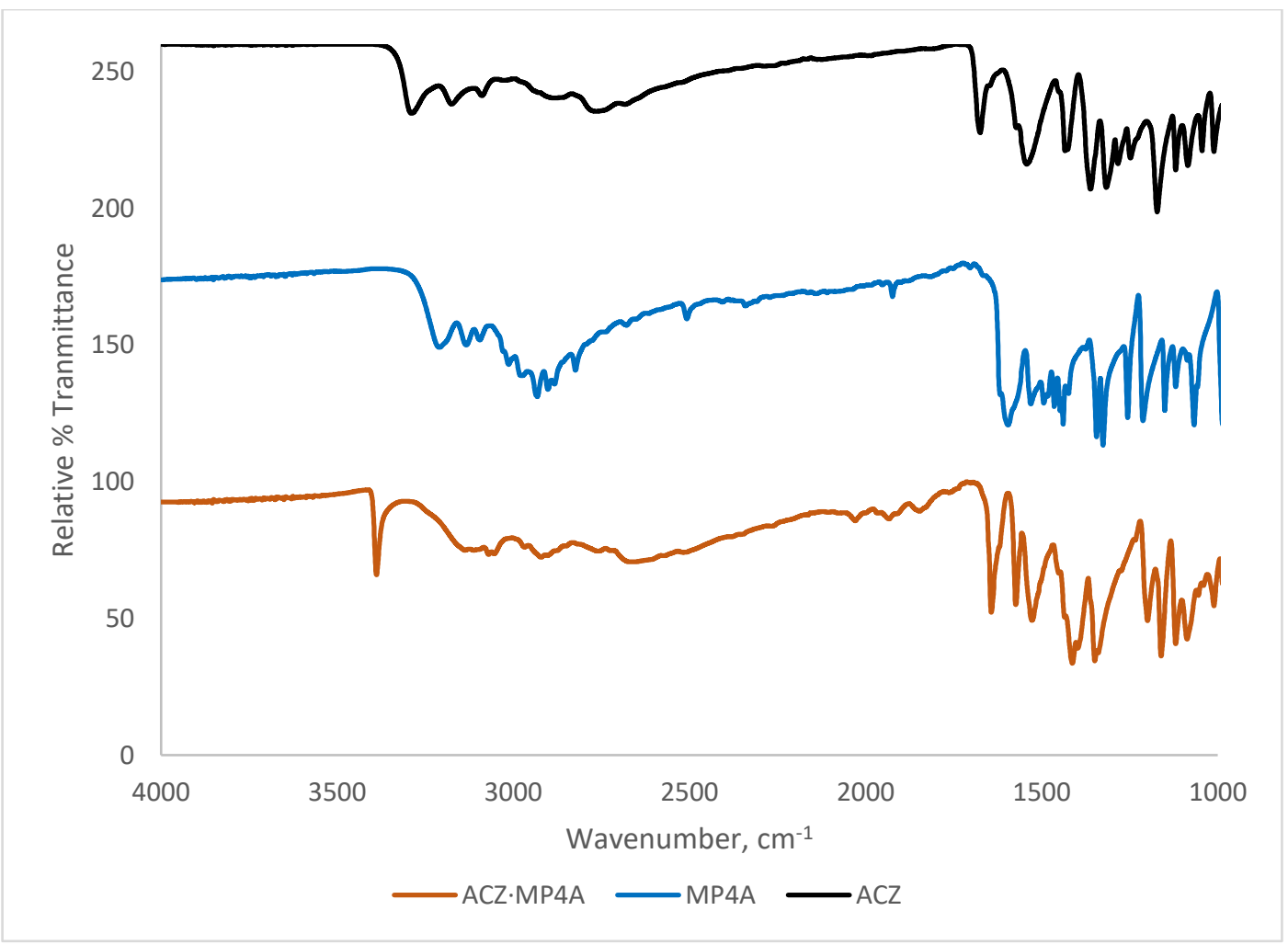

Figure S18. FTIR spectrum of ACZ, MP4A, and ionic cocrystal ACZ.MP4A. 
Table S5. Distinctive bands in the FTIR spectra for ACZ and the CCFs. Distinctive bands for the cocrystals are listed in the main text.

\begin{tabular}{|c|c|c|c|c|c|c|c|c|c|}
\hline & & \multicolumn{8}{|c|}{ CCFs } \\
\hline Band & $A C Z$ & BPEth & BPE & BIPY & 4,4'-AP & PYZ & BPP & $2 \mathrm{AP}$ & MP4A \\
\hline$v \mathrm{~N}-\mathrm{H}$ & $\begin{array}{l}3289 \\
3175 \\
3090 \\
\end{array}$ & & & & & & & $\begin{array}{l}3442 \\
3284\end{array}$ & 3210 \\
\hline$v \mathrm{C}=\mathrm{O}$ & 1675 & & & & & & & & \\
\hline $\begin{array}{c}v \mathrm{~N}-\mathrm{H}_{\|} \\
\text {(Amide II } \\
\text { band) }\end{array}$ & 1541 & & & & & & & & \\
\hline$v \mathrm{SO}_{2} \mathrm{NH}_{2}$ & $\begin{array}{l}1361 \\
1172 \\
\end{array}$ & & & & & & & & \\
\hline$v$ pyr $(\mathrm{C}=\mathrm{N})$ & & 1594 & 1593 & 1586 & 1586 & $1417^{a}$ & 1604 & 1624 & 1595 \\
\hline
\end{tabular}

a Signal for aromatic heterocycle of PYZ. 


\section{PXRD patterns}

The powder X-ray diffraction patterns were collected on a Rigaku MiniFlex II powder diffractometer. The X-ray diffraction pattern was obtained by scanning a $2 \theta$ range of $5-50^{\circ}$, step size $=0.02^{\circ}$, and scan time of $2^{\circ} / \mathrm{min}$. The $X$-ray source was Cu Ka radiation $(\lambda=1.5418 \AA)$ with an anode voltage of $30 \mathrm{kV}$ and a current of $15 \mathrm{~mA}$. Bragg-Brentano geometry was used during the data collections and the diffraction intensities were recorded on a D/teX Ultra position sensitive detector. The sample was prepared as a standard powder mount, and the diffractograms were processed through the software MDI JADE X v7.7.

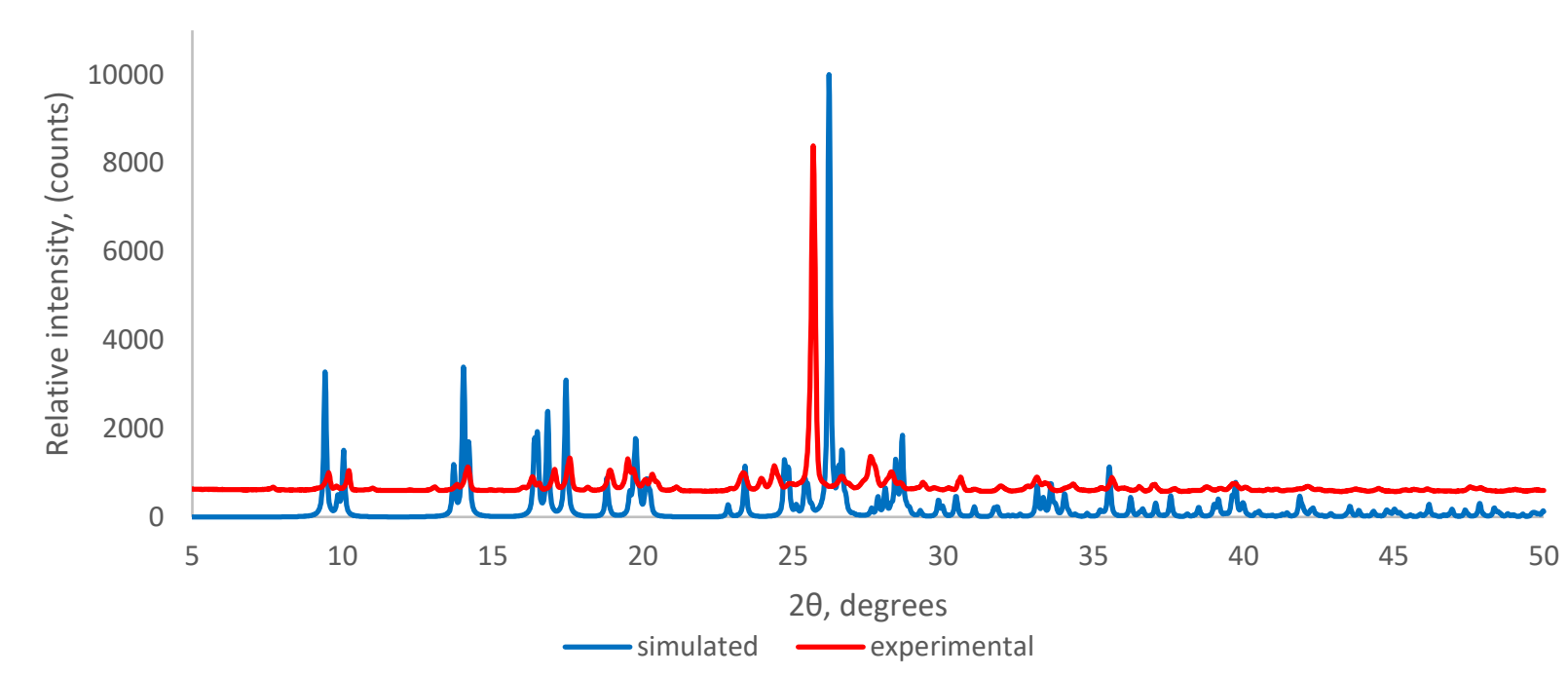

Figure S19. PXRD patterns of ACZ-BPEth: simulated from $100 \mathrm{~K}$ X-ray data and crystals synthesized by slow evaporation.

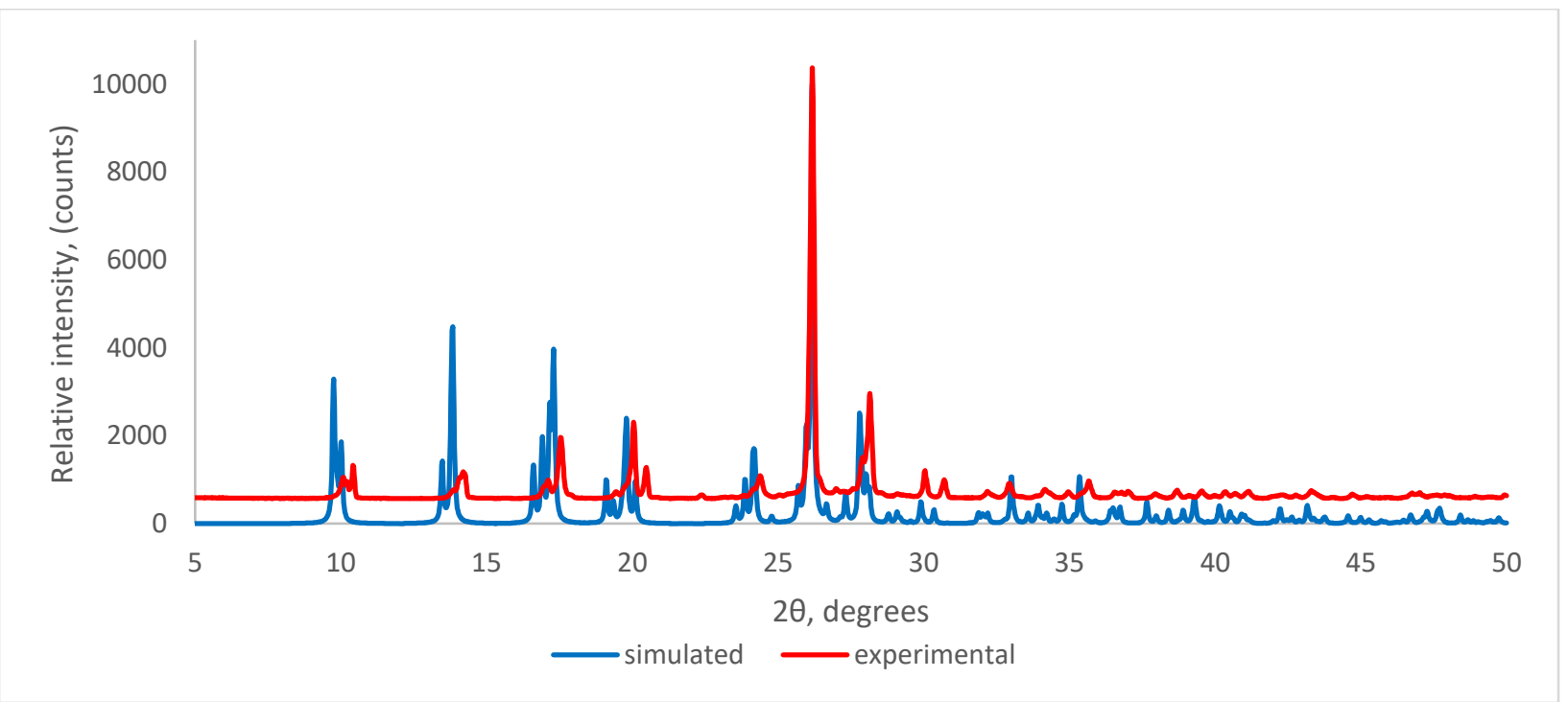

Figure S20. PXRD patterns of ACZ-BPE: simulated from $180 \mathrm{~K}$ X-ray data and crystals synthesized by slow evaporation. 


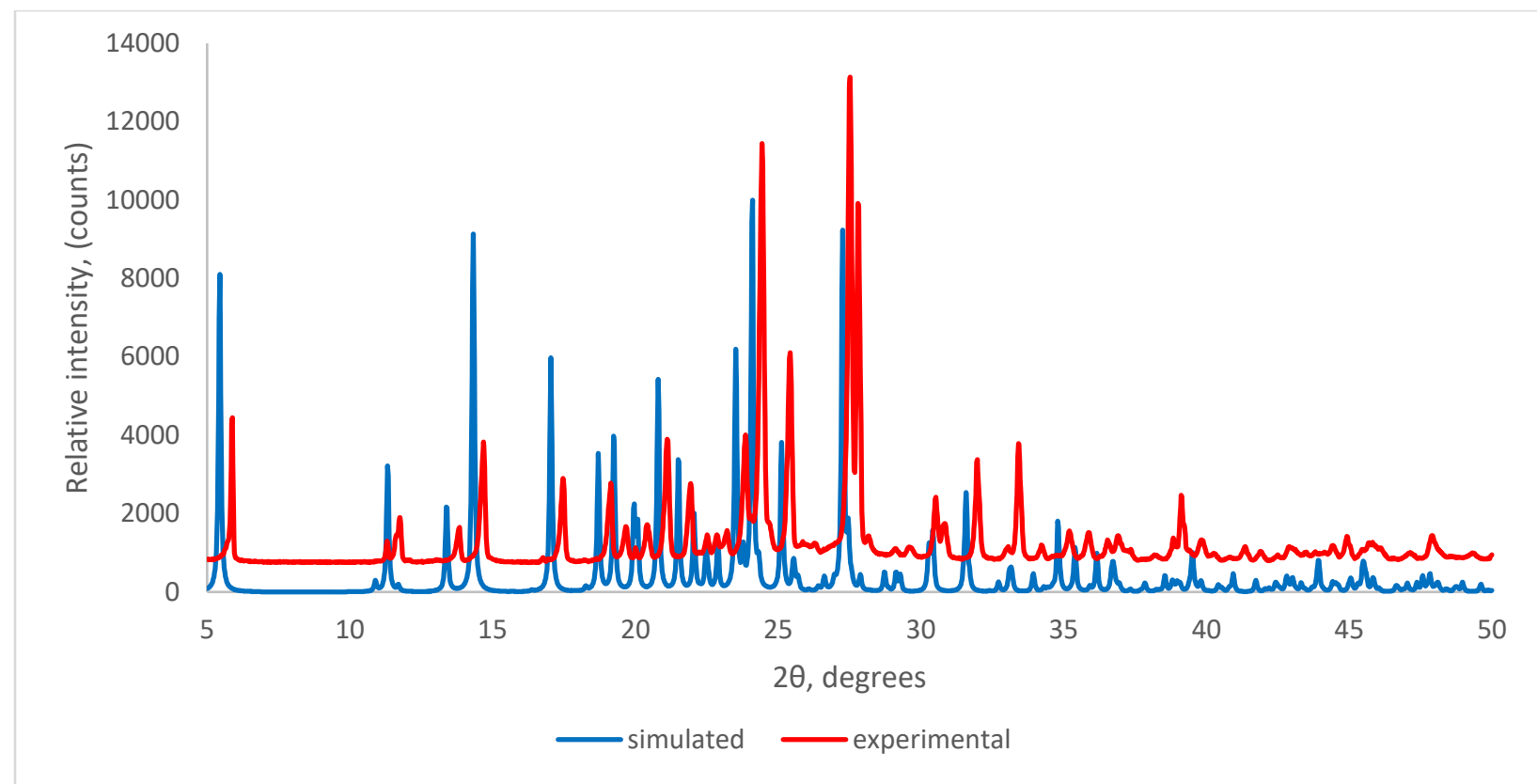

Figure S21. PXRD patterns of ACZ-BIPY: simulated from $250 \mathrm{~K}$ X-ray data and crystals synthesized by slow evaporation.

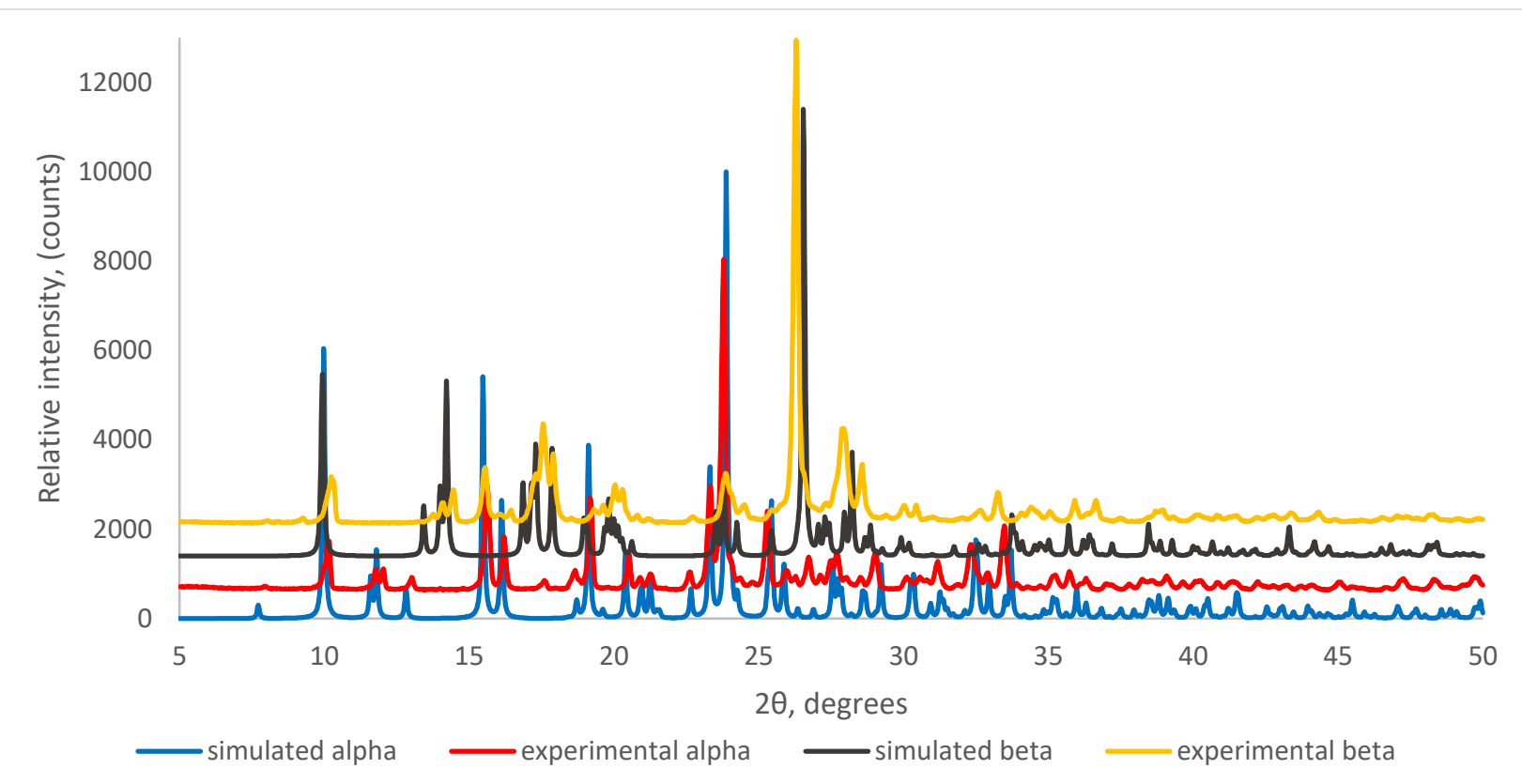

Figure S22. PXRD patterns of ACZ-4,4'-AP: simulated from $100 \mathrm{~K} \mathrm{X}$-ray data and crystals synthesized by slow evaporation. 


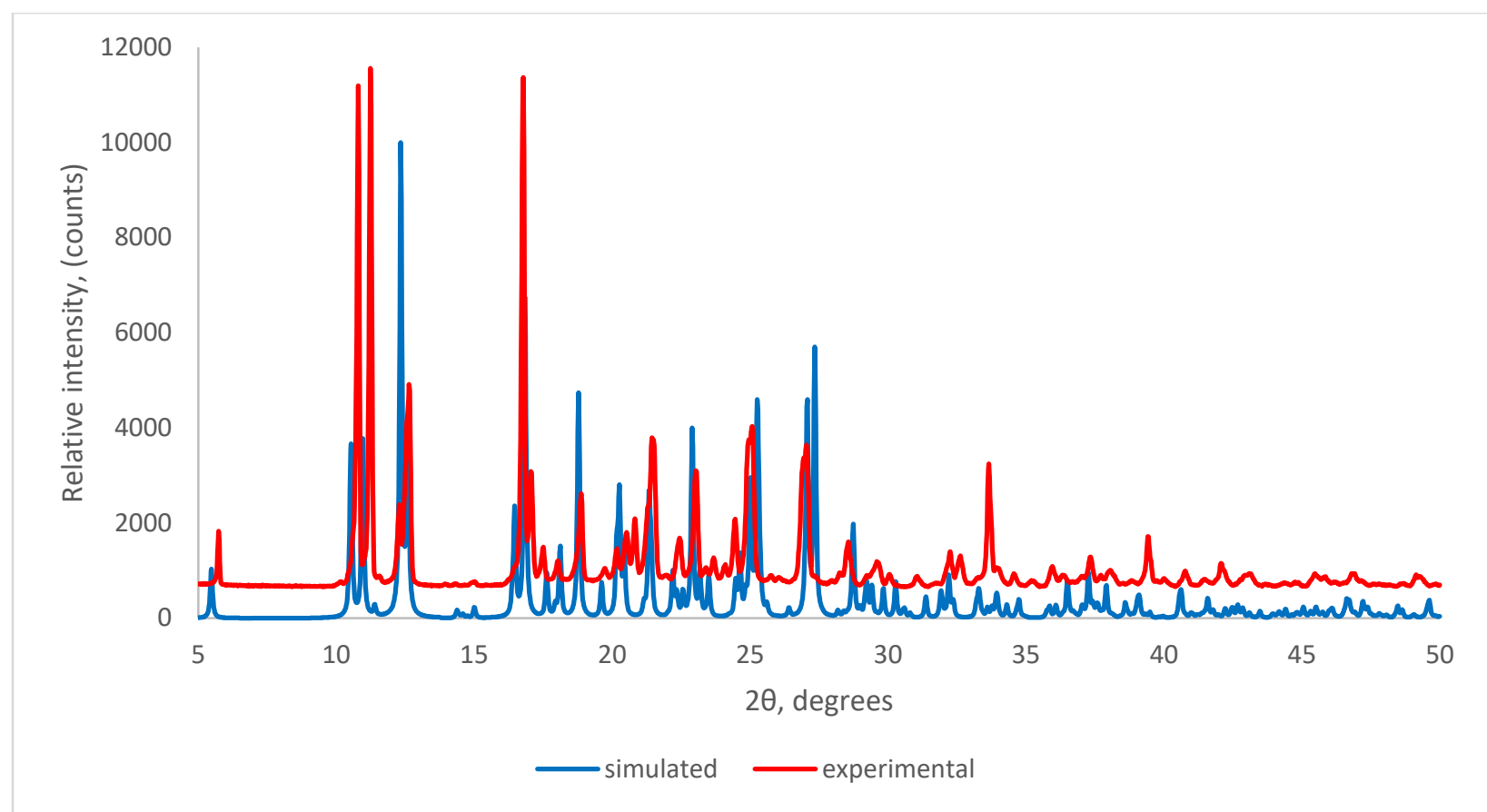

Figure S23. PXRD patterns of ACZ-BPP: simulated from $100 \mathrm{~K} \mathrm{X}$-ray data and crystals synthesized by slow evaporation.

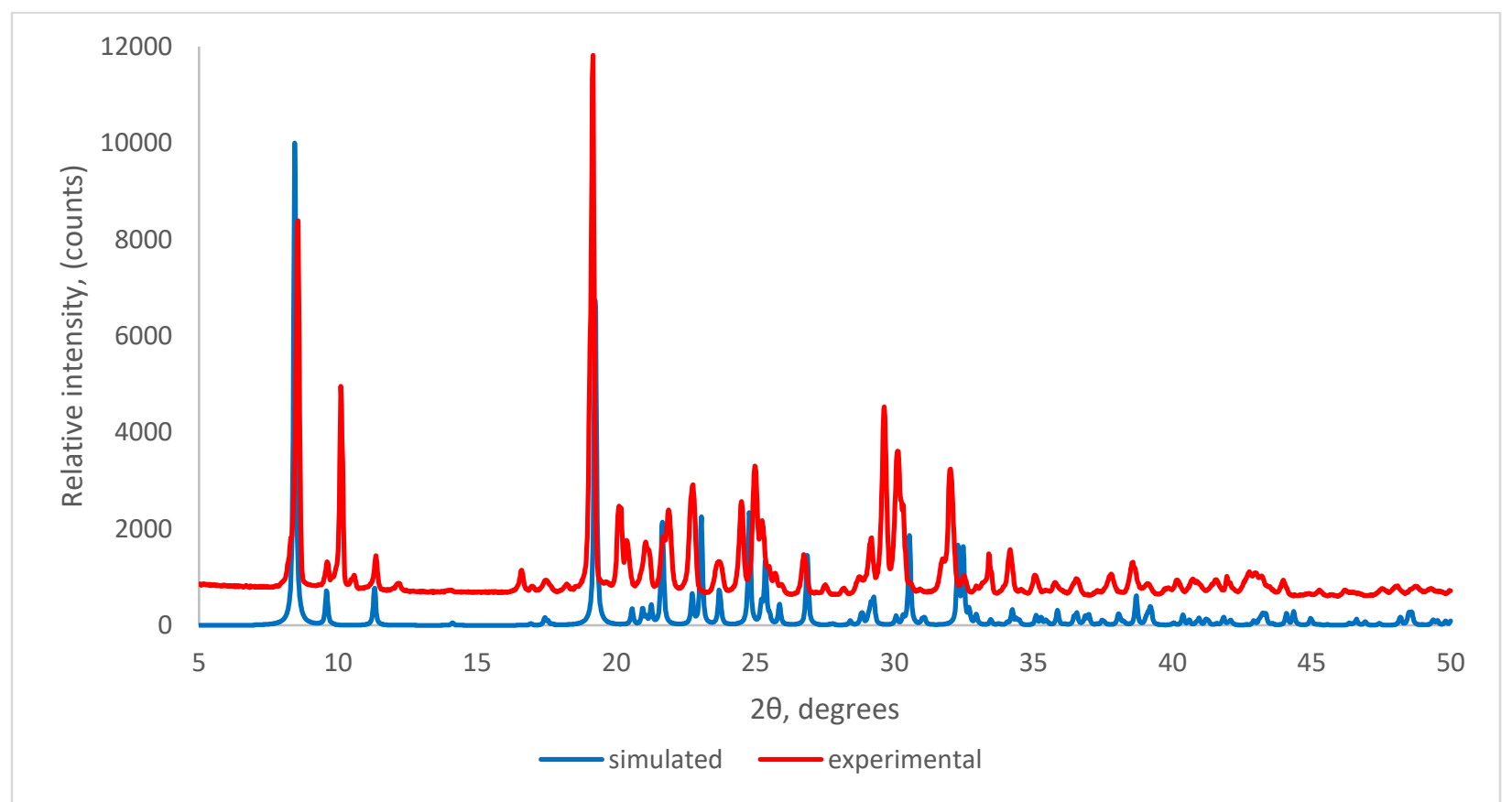

Figure S24. PXRD patterns of ACZ.PYZ: simulated from $100 \mathrm{~K} \mathrm{X}$-ray data and crystals synthesized by slow evaporation. 


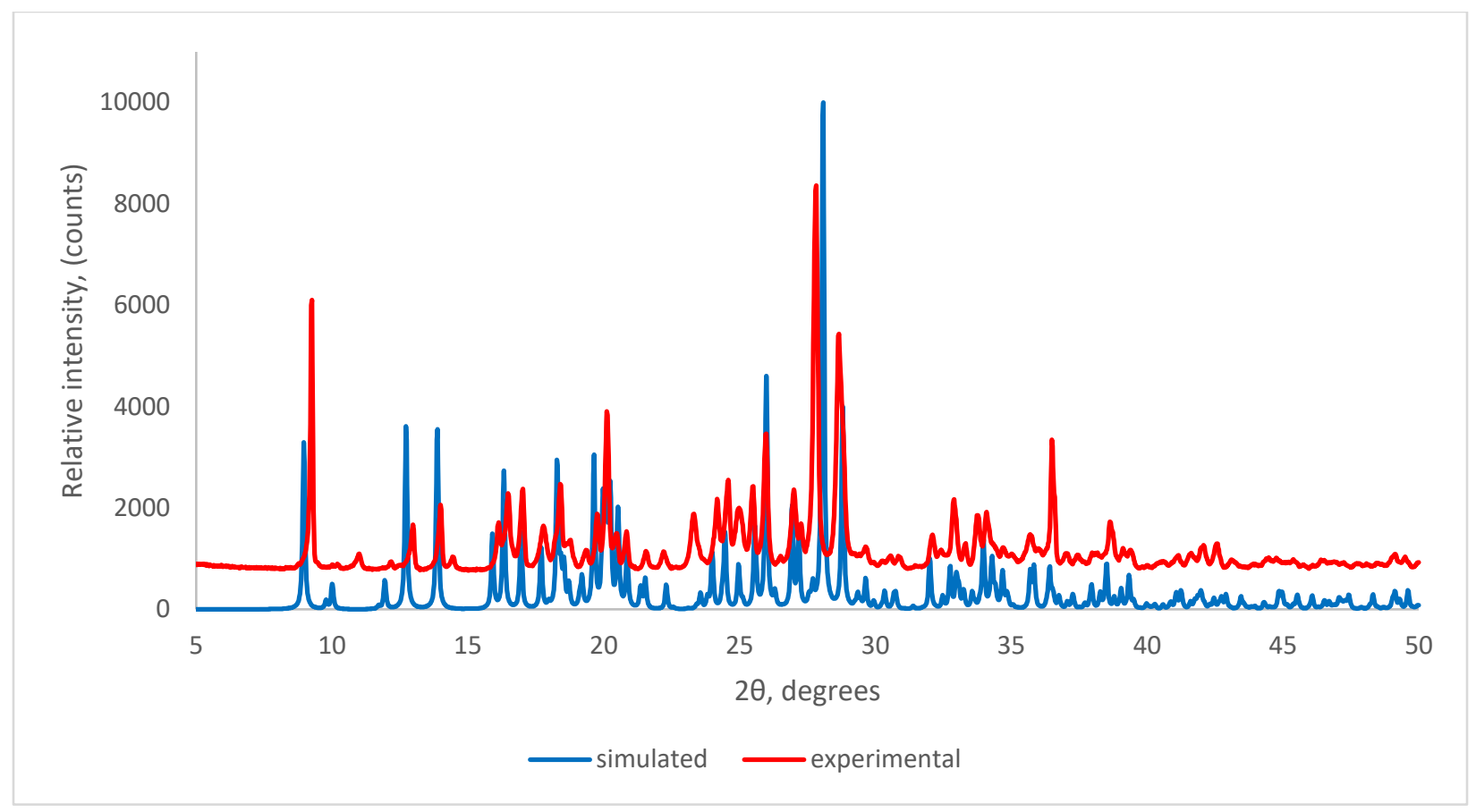

Figure S25. PXRD patterns of ACZ.2AP: simulated from $100 \mathrm{~K} \mathrm{X}$-ray data and crystals synthesized by slow evaporation.

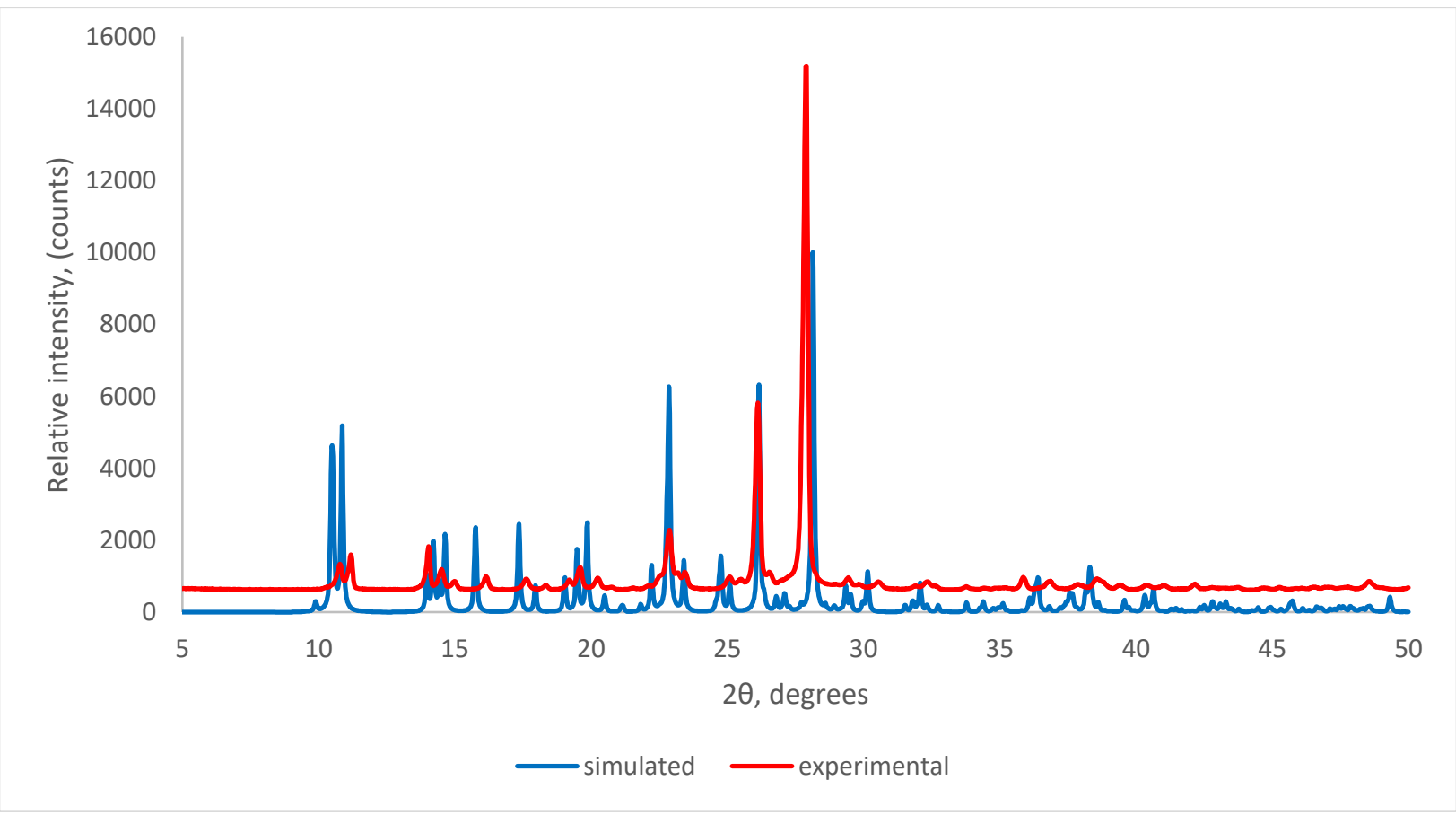

Figure S26. PXRD patterns of ACZ-MP4A: simulated from $100 \mathrm{~K}$ X-ray data and crystals synthesized by slow evaporation 


\section{Hirshfeld surface analysis}

Hirshfeld surface analysis were conducted using the program CrystalExplorer17. ${ }^{5}$ The surfaces were mapped using the $d_{\text {norm }}$ function.

(a)
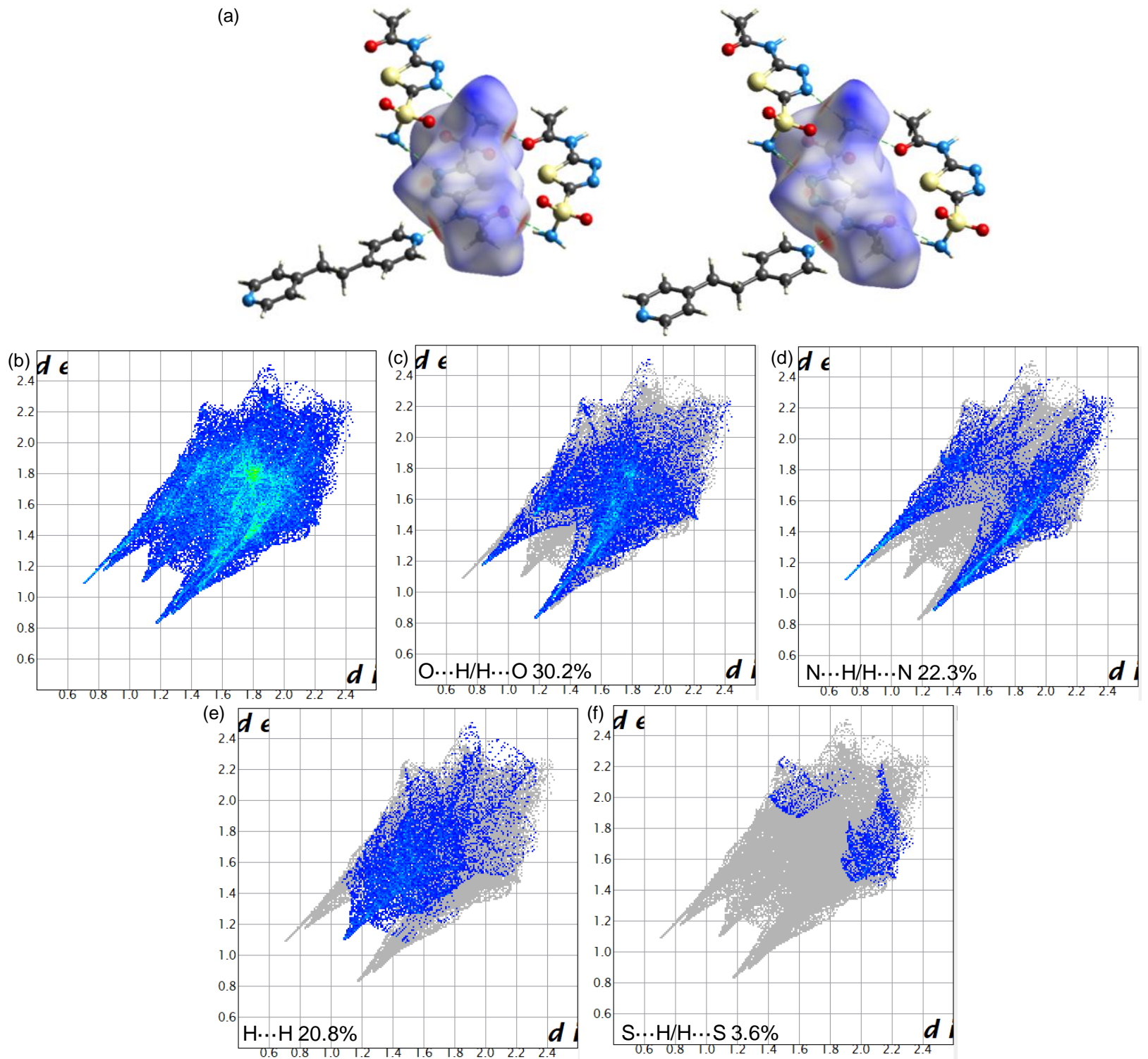

Figure S27. ACZ-BPEth: (a) Hirshfeld surface ( $\mathrm{d}_{\text {norm }}$ ) showing two views, (b) full corresponding fingerprint plot, and (c-f) decomposition of fingerprint plots into individual interactions (type denoted on plot). BPEth bonds with $\mathbf{A C Z}$ at the amide site. 
(a)
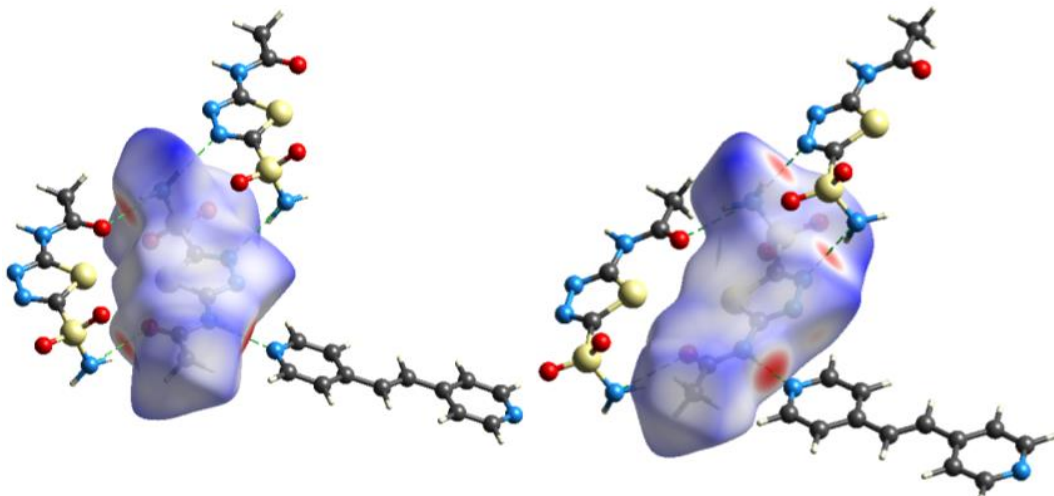

(b)
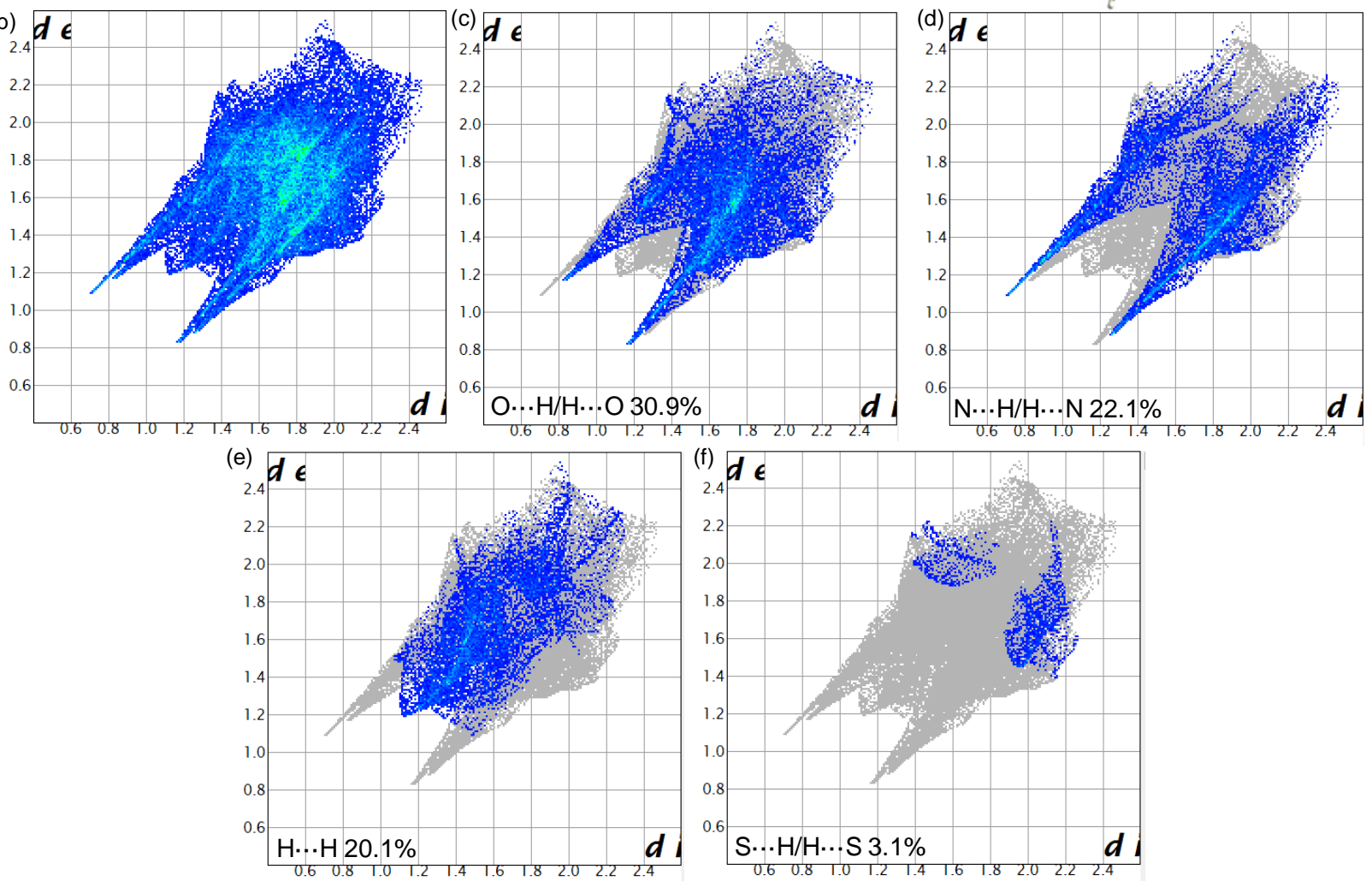

Figure S28. ACZ-BPE: (a) Hirshfeld surface ( $\mathrm{d}_{\text {norm}}$ ) showing two views, (b) full corresponding fingerprint plot, and (c-f) decomposition of fingerprint plots into individual interactions (type denoted on plot). BPE bonds with $\mathbf{A C Z}$ at the amide site. 

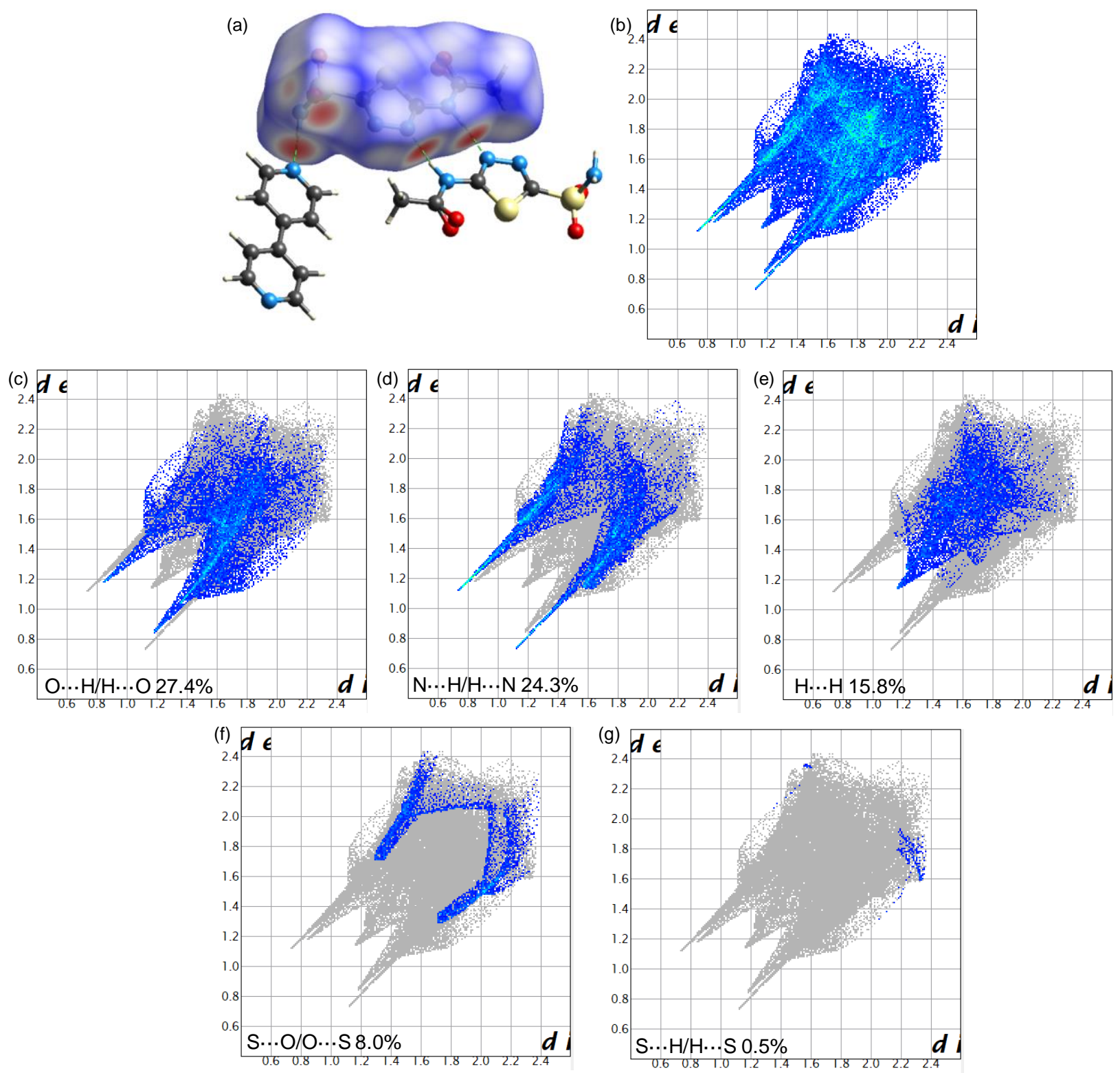

Figure S29. ACZ-BIPY: (a) Hirshfeld surface ( $\mathrm{d}_{\text {norm }}$ ), (b) full corresponding fingerprint plot, and (c-g) decomposition of fingerprint plots into individual interactions (type denoted on plot). BIPY bonds with $\mathbf{A C Z}$ at the sulfonamide site. 

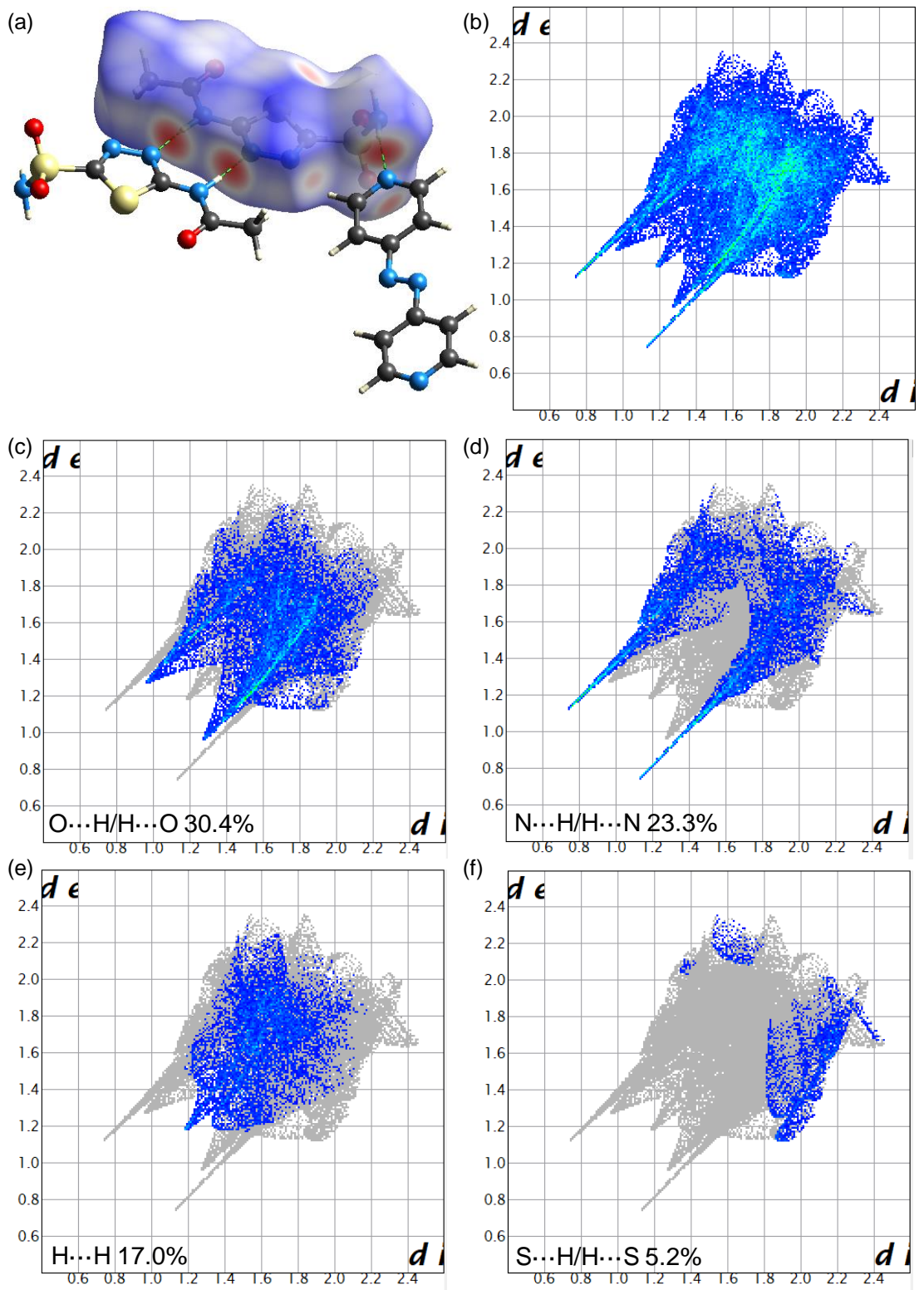

Figure S30. ACZ-4,4'-AP: (a) Hirshfeld surface ( $\mathrm{d}_{\text {norm }}$ ), (b) full corresponding fingerprint plot, and (c-f) decomposition of fingerprint plots into individual interactions (type denoted on plot). 4,4'-AP bonds with $A C Z$ at the sulfonamide site. 
(a)

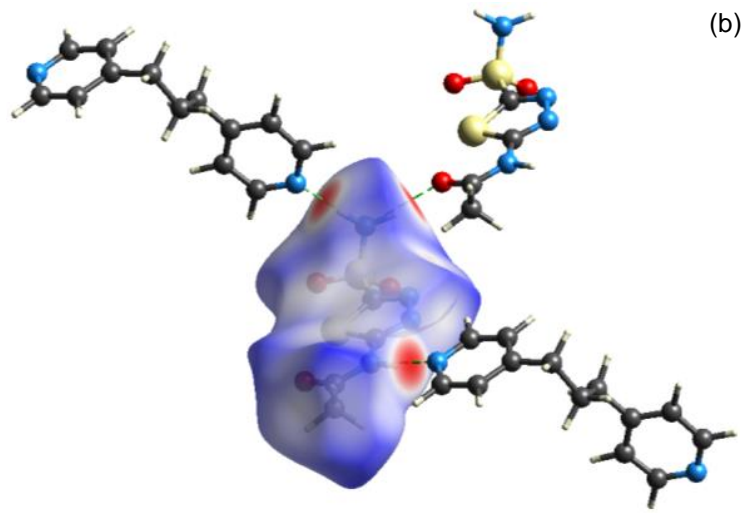

(b)

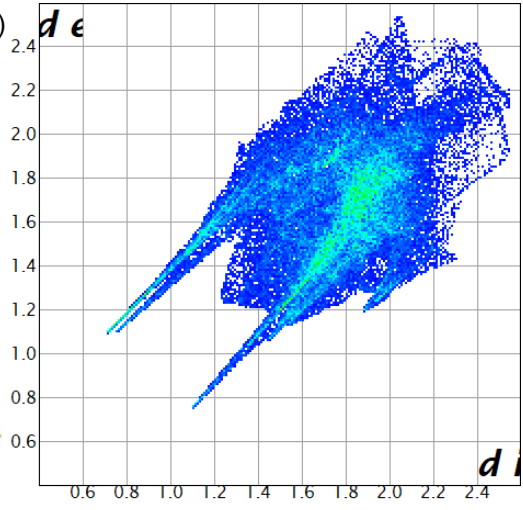

(c)
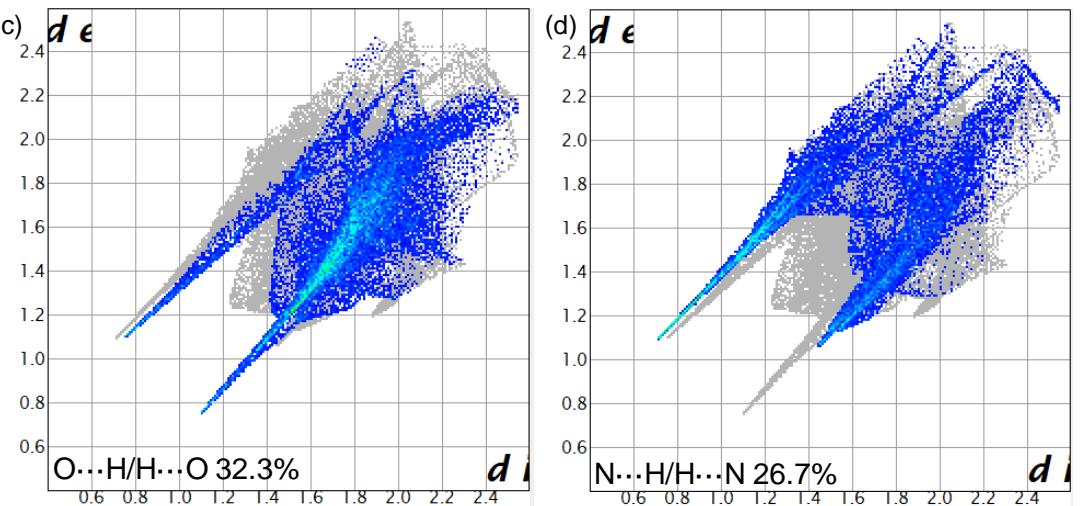

(e)
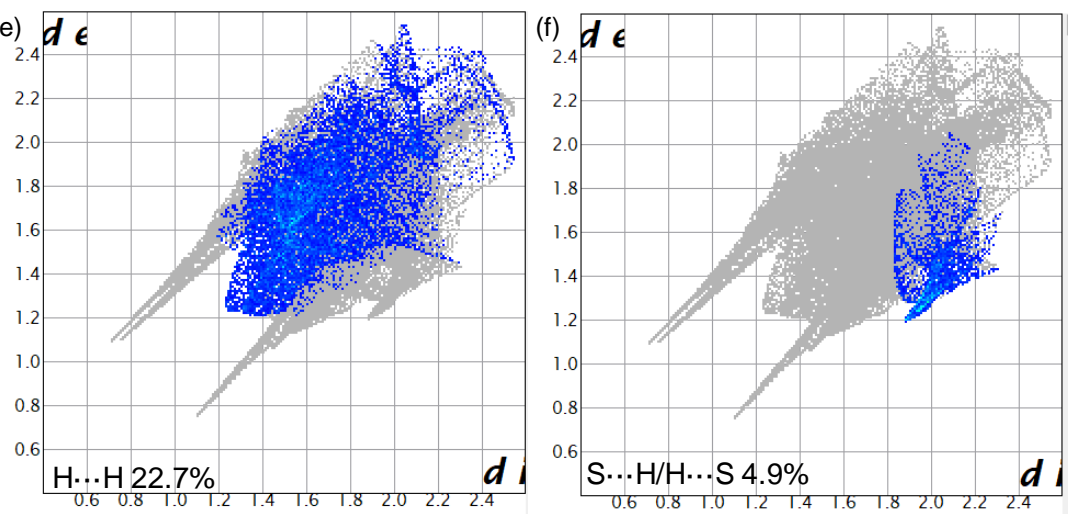

Figure S31. ACZ.BPP: (a) Hirshfeld surface ( $d_{\text {norm }}$ ), (b) full corresponding fingerprint plot, and (cf) decomposition of fingerprint plots into individual interactions (type denoted on plot). BPP bonds with $\mathbf{A C Z}$ at both the amide and sulfonamide sites. 

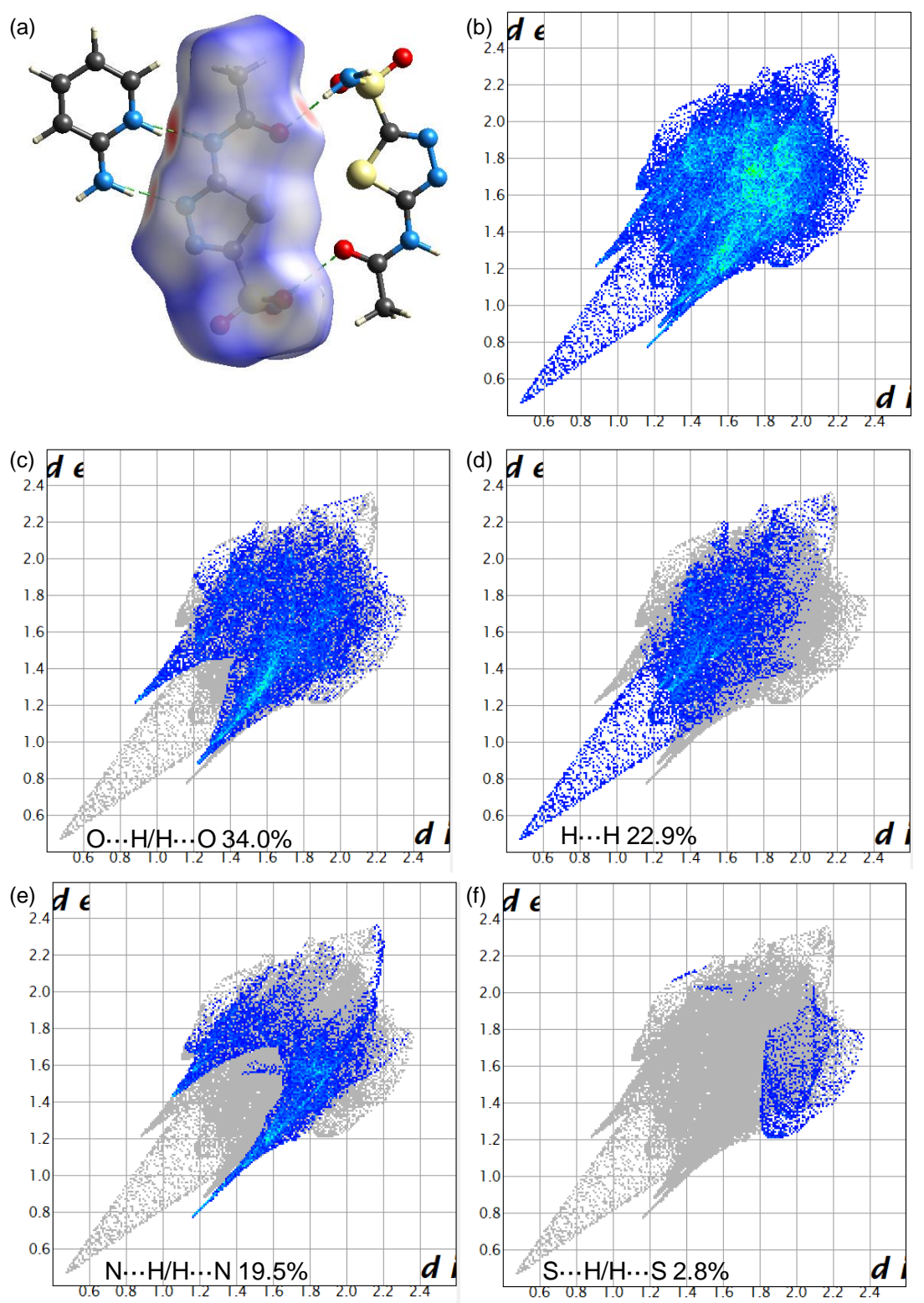

Figure S32. ACZ.2AP highlighting ACZ1 molecule that bonds with 2AP at its amide site: (a) Hirshfeld surface $\left(d_{n o r m}\right)$, (b) full corresponding fingerprint plot, and (c-f) decomposition of fingerprint plots into individual interactions (type denoted on plot). The short $\mathrm{H} \cdots \mathrm{H}$ contacts in part $\mathrm{d}$ are due to the partially transferred hydrogen between the amide and pyridine. 
(a)

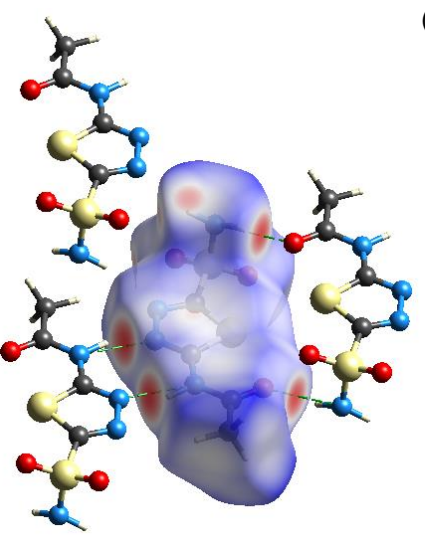

(c)

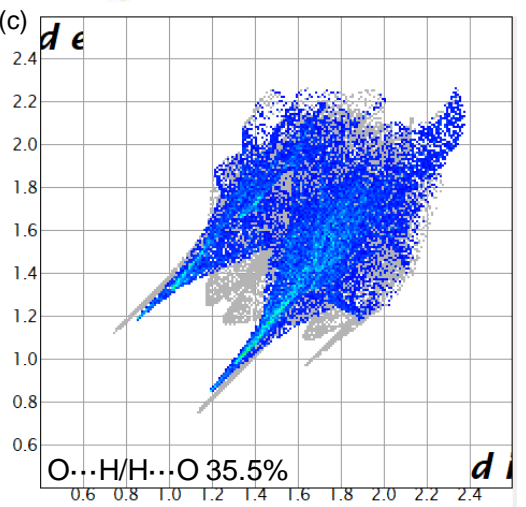

(e)

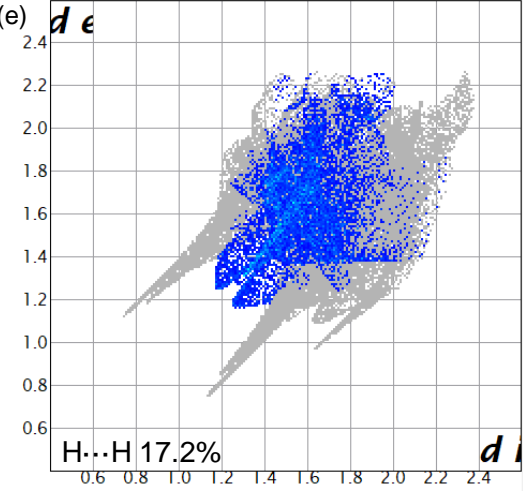

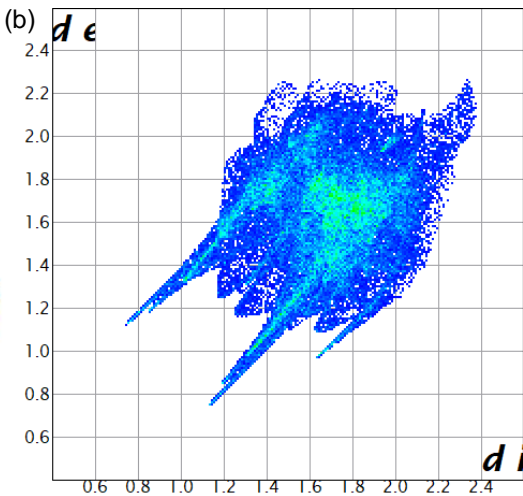
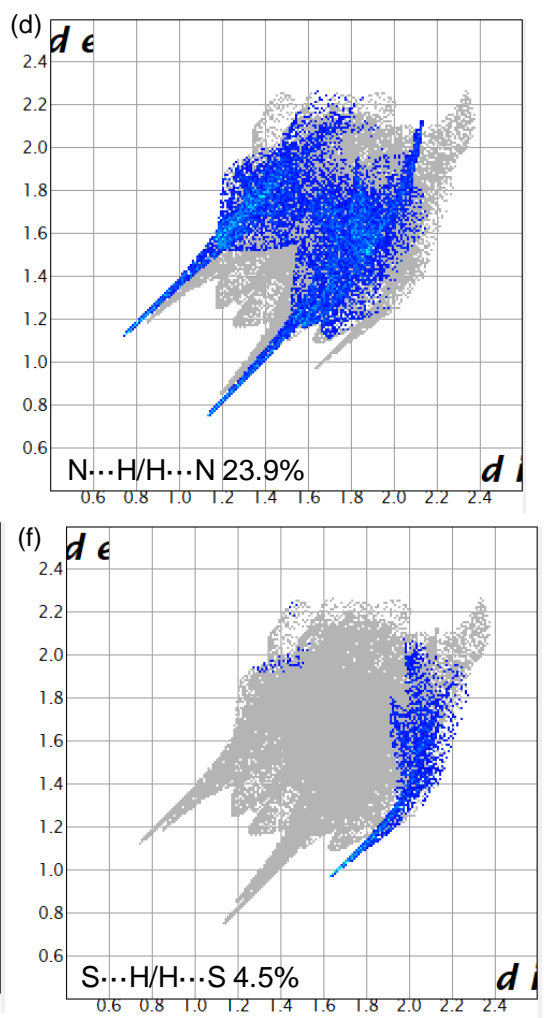

Figure S33. ACZ-2AP highlighting ACZ2 that only bonds with other ACZ molecules: (a) Hirshfeld surface $\left(\mathrm{d}_{\text {norm }}\right)$, (b) full corresponding fingerprint plot, and (c-f) decomposition of fingerprint plots into individual interactions (type denoted on plot). 
(a)

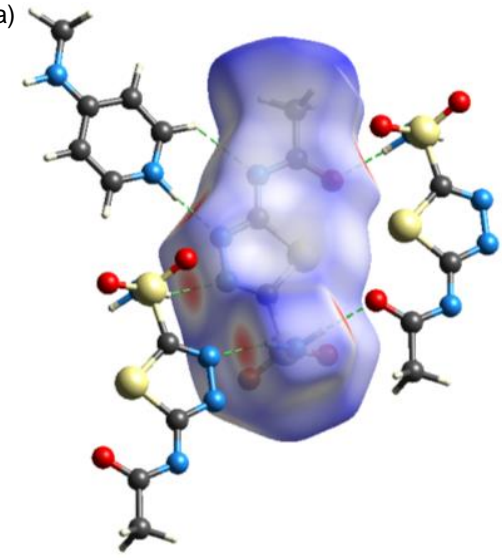

(c)

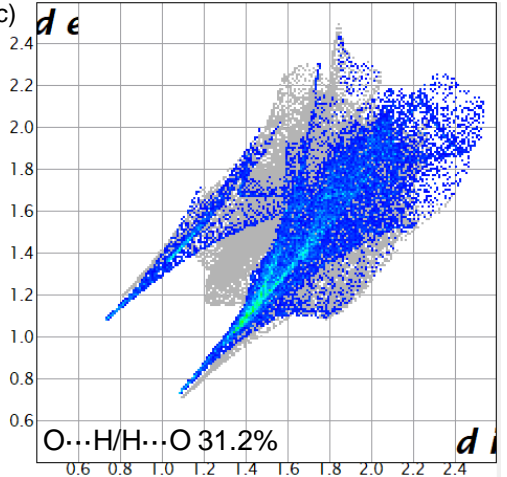

(e)

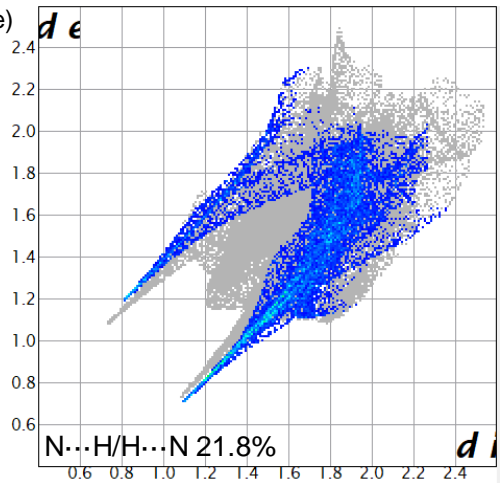

(b)
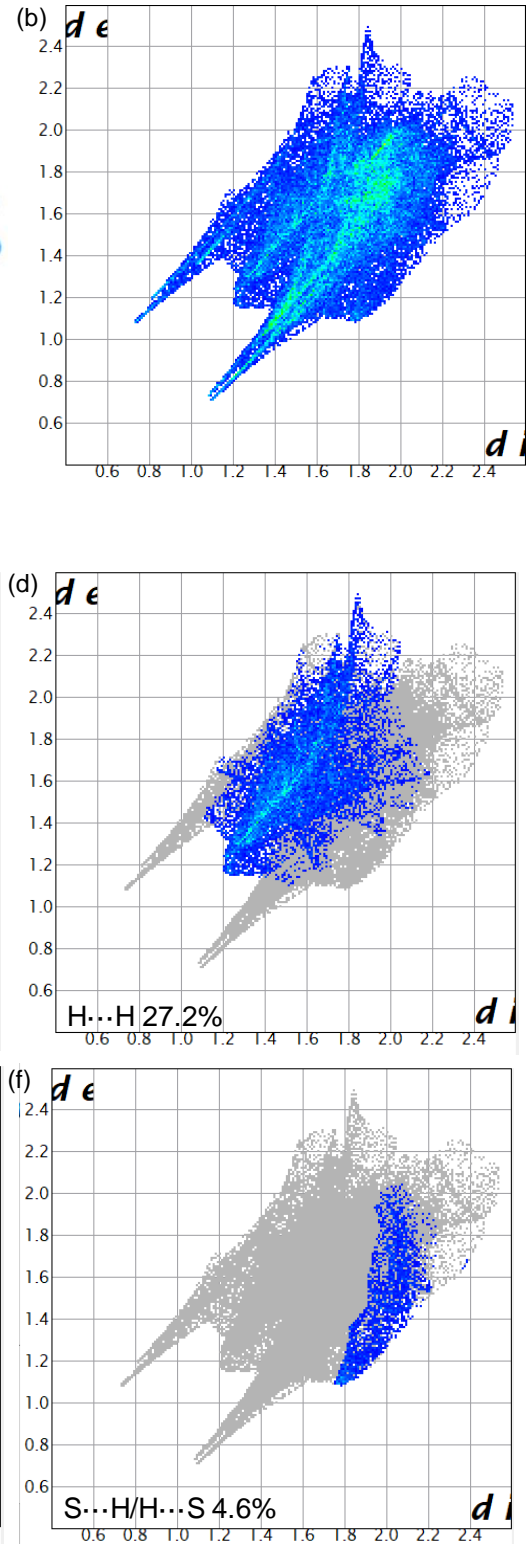

Figure S34. ACZ-MP4A: (a) Hirshfeld surface ( $d_{n o r m}$ ), (b) full corresponding fingerprint plot, and (c-f) decomposition of fingerprint plots into individual interactions (type denoted on plot). MP4A bonds with $\mathrm{ACZ}$ at the amide site. 


\section{X-ray crystal structure}

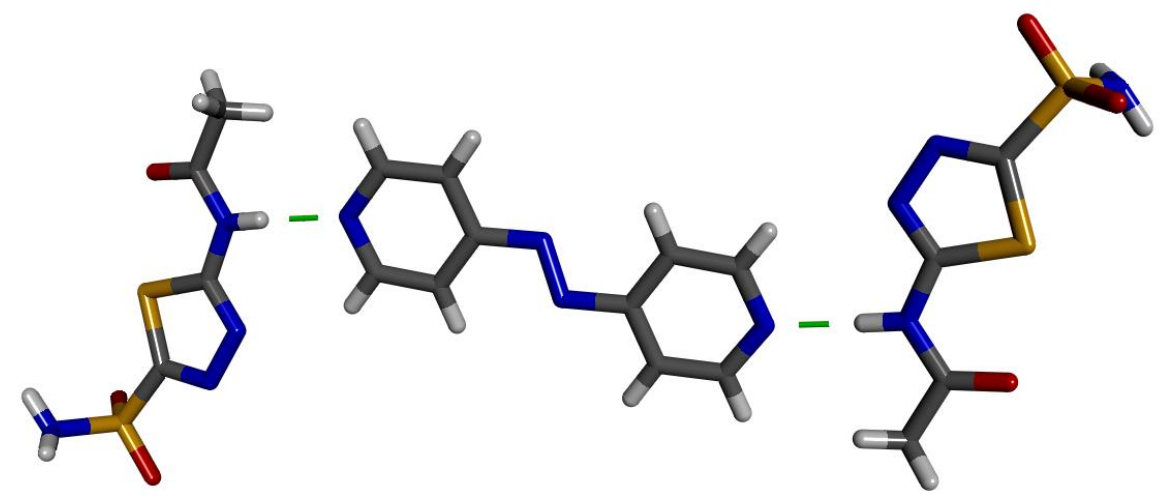

Figure S35. X-ray crystal structure of ACZ-4,4'-AP beta polymorph.

\section{References}

1. Krause et al., (2015) SADABS v 2016/2.

2. Sheldrick, G. M. Acta Crystallogr., Sect. C: Cryst. Struct. Commun., 2015, C71, 3-8.

3. CrysAlis ${ }^{\text {Pro }}$ (2018) Oxford Diffraction Ltd.

4. SCALE3 ABSPACK (2005) Oxford Diffraction Ltd.

5. Turner, M. J.; McKinnon, J. J.; Wolff, S. K.; Grimwood, D. J.; Spackman, P. R.; Jayatilaka D.; Spackman, M. A. CrystalExplorer17 (2017). University of Western Australia. http://hirshfeldsurface.net 